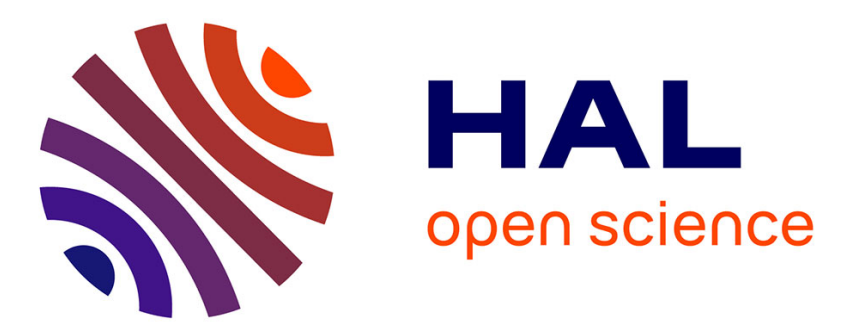

\title{
An affine formulation for the prediction of the effective properties of nonlinear composites and polycrystals
}

\author{
Renaud Masson, Michel Bornert, P. Suquet, A. Zaoui
}

\section{To cite this version:}

Renaud Masson, Michel Bornert, P. Suquet, A. Zaoui. An affine formulation for the prediction of the effective properties of nonlinear composites and polycrystals. Journal of the Mechanics and Physics of Solids, 2000, 48 (6-7), pp.1203-1227. 10.1016/S0022-5096(99)00071-X . hal-03427478

\section{HAL Id: hal-03427478 \\ https://hal.science/hal-03427478}

Submitted on 13 Dec 2021

HAL is a multi-disciplinary open access archive for the deposit and dissemination of scientific research documents, whether they are published or not. The documents may come from teaching and research institutions in France or abroad, or from public or private research centers.
L'archive ouverte pluridisciplinaire HAL, est destinée au dépôt et à la diffusion de documents scientifiques de niveau recherche, publiés ou non, émanant des établissements d'enseignement et de recherche français ou étrangers, des laboratoires publics ou privés. 


\title{
An affine formulation for the prediction of the effective properties of nonlinear composites and polycrystals
}

\author{
R. Masson ${ }^{\mathrm{a}}$, M. Bornert ${ }^{\mathrm{a}}$, P. Suquet ${ }^{\mathrm{b}}$, A. Zaoui ${ }^{\mathrm{a}, *}$ \\ ${ }^{a}$ Laboratoire de Mécanique des Solides, CNRS-École Polytechnique, 91128 Palaiseau Cedex, France \\ ${ }^{\mathrm{b}}$ Laboratoire de Mécanique et d'Acoustique, CNRS, Marseille, France
}

Received 10 February 1999; received in revised form 20 July 1999

\begin{abstract}
Variational approaches for nonlinear elasticity show that Hill's incremental formulation for the prediction of the overall behaviour of heterogeneous materials yields estimates which are too stiff and may even violate rigorous bounds. This paper aims at proposing an alternative 'affine' formulation, based on a linear thermoelastic comparison medium, which could yield softer estimates. It is first described for nonlinear elasticity and specified by making use of Hashin-Shtrikman estimates for the linear comparison composite; the associated affine self-consistent predictions are satisfactorily compared with incremental and tangent ones for power-law creeping polycrystals. Comparison is then made with the second-order procedure (Ponte Castañeda, P., 1996. Exact second-order estimates for the effective mechanical properties of nonlinear composite materials. J. Mech. Phys. Solids, 44 (6), 827-862) and some limitations of the affine method are pointed out; explicit comparisons between different procedures are performed for isotropic, two-phase materials. Finally, the affine formulation is extended to history-dependent behaviours; application to the self-consistent modelling of the elastoplastic behaviour of polycrystals shows that it offers an improved alternative to Hill's incremental formulation. (C) 2000 Elsevier Science Ltd. All rights reserved.
\end{abstract}

Keywords: Inhomogeneous material; Polycrystalline material; Porous material; Viscoplastic material; Homogenisation

\footnotetext{
* Corresponding author.
} 


\section{Introduction}

Nonlinear estimates for the effective properties of heterogeneous materials were first proposed for predicting the elastoplastic behaviour of polycrystals. The initial contributions of the Taylor (1938) type, as well as those using an elastic description of the internal interactions between the crystals (Kröner, 1961), make no recourse to any linearisation procedure. Hill's (1965) incremental formulation of the elastoplastic self-consistent scheme is based on the use of instantaneous or 'tangent' (multibranched) moduli or compliances relating the stress and strain rate tensors at both the local and the global scales within a step-by-step homogenisation procedure. It is the actual starting point of a whole range of schemes using linearisation of the constitutive relations of the phases. This incremental formulation has been considered for a long time the standard for deriving nonlinear estimates, especially for 'hereditary' behaviours, i.e., for those behaviours which exhibit dependence of the current response on the loading path. However, an incremental formulation is no longer necessary for non-hereditary behaviours such as steady-state creep. Hutchinson (1976) extended Hill's procedure to this case by using tangent creep compliances instead of elastoplastic ones. He noticed that, for power-law creep, Hill's formulation may be integrated into a 'total' one making use of 'secant' creep compliances. Other secant moduli or compliances have also been defined (Berveiller and Zaoui, 1979; Tandon and Weng, 1988) leading to a (classical) 'secant formulation', closely related to Hill's incremental formulation.

The development of variational approaches (Willis, 1983; Talbot and Willis, 1985; Ponte Castañeda, 1991; Suquet, 1993) for behaviours deriving from a single potential (nonlinear elasticity or viscosity) has made it possible to compare some of these nonlinear estimates to rigorous bounds of Hashin-Shtrikman type. A general conclusion (Gilormini, 1996) is that both the incremental and the (classical) secant formulations lead to estimates that are too 'stiff' and can even violate these bounds in some cases. The main reason for this is that the local tangent or secant moduli, which should vary from point to point, even inside a given phase, are assumed to be piecewise uniform (i.e., uniform per phase) and defined at some reference stress or strain which, as a rule, is taken to be the average stress or strain per phase.

Two different routes have been explored in order to overcome this difficulty. On the one hand, several modifications have been brought into the secant approach to take better into account the stress (or strain) fluctuations within the individual phases. A first attempt was made for particulate composites by Qiu and Weng (1992). It consists in using the average shear energy in the matrix to define its effective stress. However, the result is valid only for incompressible materials. A more general theory, valid for arbitrary microstructures and independent of any assumption of phase incompressibility, has been proposed by Suquet (1995) (see also Suquet, 1997). It is based on second-order moments in each individual phase of the linear comparison solid. The use of second-order moments has also been considered independently by $\mathrm{Hu}$ (1996) and Buryachenko (1996). Interestingly, it 
has been shown (Suquet, 1995; Ponte Castañeda and Suquet, 1998) that this secant theory based on second-order moments coincides with Ponte Castañeda's (1991) variational procedure; this property ensures that the resulting estimates do not lead to the violation of any bound. On the other hand, new linearisations have been searched for, with the objective of generating softer estimates even when using the classical reference quantities. This is the case for the 'second-order procedure' proposed by Ponte Castañeda (1996) which makes use of a secondorder Taylor development of the strain or stress potentials. This paper aims at proposing an alternative solution, the 'affine formulation', initially suggested by Rougier et al. (1994), which makes use of the stress-strain relations instead of the potentials; we will discuss both its limitations and its advantages with respect to other procedures.

We first define this affine formulation for nonlinear elasticity or viscosity (Section 2), with an application to the self-consistent scheme in view of a comparison with the incremental formulation as well as with the tangent formulation proposed by Molinari et al. (1987) for power-law creep. Next, we compare the affine formulation with the second-order procedure and we extend this comparison to other schemes, specifically for two-phase composites (Section 3). Finally, we apply the affine formulation to hereditary behaviours, with special emphasis on crystalline elastoplasticity for a direct comparison with Hill's formulation (Section 4).

\section{The affine formulation for nonlinear elasticity or viscosity}

\subsection{Principle of the affine formulation}

An incremental formulation is not required for nonlinear elasticity and viscosity. Therefore, the tangent moduli or compliances, associated with some given initial prestress or prestrain (or prestrain rate), could give, at any stage, a better description of the current local behaviour than any secant ones. We deal here with nonlinear elasticity, but all the forthcoming developments are still valid for viscous materials with $\boldsymbol{\varepsilon}(\boldsymbol{x})$ denoting the strain rate. We consider a given deformed state (0) of a representative volume element $\Omega$ of the heterogeneous material under consideration. At this stage, let $\boldsymbol{\varepsilon}^{(0)}(\boldsymbol{x})$ and $\boldsymbol{\sigma}^{(0)}(\boldsymbol{x})$ be the strain and stress tensors at some point $\boldsymbol{x}$ in some phase $s$. The nonlinear stress-strain relations for this phase read $\boldsymbol{\sigma}=\boldsymbol{H}_{s}(\boldsymbol{\varepsilon})$ or equivalently $\boldsymbol{\varepsilon}=\boldsymbol{G}_{s}(\boldsymbol{\sigma})$. Their linearised 'affine' approximation at this stage (0) reads at $\boldsymbol{x}$

$$
\boldsymbol{\sigma}=\boldsymbol{L}_{s}^{(0)}(\boldsymbol{x}): \boldsymbol{\varepsilon}+\boldsymbol{\tau}^{(0)}(\boldsymbol{x}), \quad \text { or, equivalently, } \quad \boldsymbol{\varepsilon}=\boldsymbol{M}_{s}^{(0)}(\boldsymbol{x}): \boldsymbol{\sigma}+\boldsymbol{\eta}^{(0)}(\boldsymbol{x})
$$

with

$$
\boldsymbol{L}_{s}^{(0)}(\boldsymbol{x})=\frac{\mathrm{d} H_{s}}{\mathrm{~d} \boldsymbol{\varepsilon}}\left(\varepsilon^{(0)}(\boldsymbol{x})\right), \quad \boldsymbol{\tau}^{(0)}(\boldsymbol{x})=\boldsymbol{H}_{s}\left(\boldsymbol{\varepsilon}^{(0)}(\boldsymbol{x})\right)-\boldsymbol{L}_{s}^{(0)}(\boldsymbol{x}): \varepsilon^{(0)}(\boldsymbol{x})
$$




$$
\boldsymbol{M}_{s}^{(0)}(\boldsymbol{x})=\frac{\mathrm{d} \boldsymbol{G}_{s}}{\mathrm{~d} \boldsymbol{\sigma}}\left(\boldsymbol{\sigma}^{(0)}(\boldsymbol{x})\right), \quad \boldsymbol{\eta}^{(0)}(\boldsymbol{x})=\boldsymbol{G}_{s}\left(\boldsymbol{\sigma}^{(0)}(\boldsymbol{x})\right)-\boldsymbol{M}_{s}^{(0)}(\boldsymbol{x}): \boldsymbol{\sigma}^{(0)}(\boldsymbol{x}) .
$$

The tensors $\boldsymbol{L}_{s}^{(0)}$ and $\boldsymbol{M}_{s}^{(0)}$ are reciprocals of each other and exhibit diagonal symmetry due to the existence of a stress or strain potential (see Section 3 ). When this procedure is applied throughout $\Omega$, the nonlinear-elastic heterogeneous body is transformed into a linear thermoelastic body (by identifying the prestrain or prestress tensors with their thermal analogues). This 'linear thermoelastic comparison composite' is a continuously heterogeneous body, i.e. a composite with infinitely many phases. Assume for a while that we can deal with this difficulty in the purely elastic case (no prestrain or prestress field) and derive the corresponding strain and stress localisation tensor fields $\boldsymbol{A}_{s}^{(0)}(\boldsymbol{x})$ and $\boldsymbol{B}_{s}^{(0)}(\boldsymbol{x})$. They can be used to get the overall constitutive equations of the thermoelastic medium by:

$$
\begin{aligned}
& \overline{\boldsymbol{\sigma}}=\tilde{\boldsymbol{L}}^{(0)}: \overline{\boldsymbol{\varepsilon}}+\tilde{\boldsymbol{\tau}}^{(0)}, \quad \tilde{\boldsymbol{L}}^{(0)}=\left\langle\boldsymbol{L}^{(0)}: \boldsymbol{A}^{(0)}, \quad \tilde{\boldsymbol{\tau}}^{(0)}=\left\langle\boldsymbol{\tau}^{(0)}: \boldsymbol{A}^{(0)}\right.\right. \\
& \overline{\boldsymbol{\varepsilon}}=\tilde{\boldsymbol{M}}^{(0)}: \overline{\boldsymbol{\sigma}}+\tilde{\boldsymbol{\eta}}^{(0)}, \quad \tilde{\boldsymbol{M}}^{(0)}=\left\langle\boldsymbol{M}^{(0)}: \boldsymbol{B}^{(0)}, \quad \tilde{\boldsymbol{\eta}}^{(0)}=\left\langle\boldsymbol{\eta}^{(0)}: \boldsymbol{B}^{(0)}\right.\right.
\end{aligned}
$$

where $\langle\cdot\rangle$ indicates a spatial average. These equations can only be used when the strain or stress fields $\boldsymbol{\varepsilon}^{(0)}(\boldsymbol{x})$ or $\boldsymbol{\sigma}^{(0)}(\boldsymbol{x})$ are known. They have to be combined with strain or stress localisation equations for the thermoelastic problem (which do not derive, in the general case, from the knowledge of $\boldsymbol{A}_{s}^{(0)}(\boldsymbol{x})$ and $\left.\boldsymbol{B}_{s}^{(0)}(\boldsymbol{x})\right)$; this results in a global set of implicit equations for $\boldsymbol{\varepsilon}^{(0)}(\boldsymbol{x})$ or $\boldsymbol{\sigma}^{(0)}(\boldsymbol{x})$

\subsection{Approximation of piecewise uniformity}

Such a treatment is not practical and one has, as usual, to adopt the simplification of piecewise uniform moduli and prestresses (compliances and prestrains) per phase. This is done by referring these quantities to some reference strain $\boldsymbol{\varepsilon}_{s}$ (stress $\boldsymbol{\sigma}_{s}$ ) in every phase. This choice is an essential one. To make clearer the specific aspects of the affine approach with respect to other classical formulations, we prescribe, as do those, the reference strain (stress) to be the average per phase of the strain (stress) field. The procedure is detailed for prestrains and compliances; dual results are obtained upon appropriate substitution of the relevant fields and variables.

Thus, at any stage, every phase $s$ is supposed to obey the linearised constitutive equations (for clarity, the index 0 referring to the current stage is omitted):

$$
\boldsymbol{\varepsilon}=\boldsymbol{M}_{s}: \boldsymbol{\sigma}+\boldsymbol{\eta}_{s}, \quad \boldsymbol{M}_{s}=\frac{\mathrm{d} \boldsymbol{G}_{s}}{\mathrm{~d} \boldsymbol{\sigma}}\left(\boldsymbol{\sigma}_{s}\right), \quad \boldsymbol{\eta}_{s}=\boldsymbol{G}_{s}\left(\boldsymbol{\sigma}_{s}\right)-\boldsymbol{M}_{s}: \boldsymbol{\sigma}_{s} .
$$

We emphasise that the approximate fields $\boldsymbol{\varepsilon}$ and $\boldsymbol{\sigma}$ in Eq. (4) are different from the actual fields in the nonlinear composite. They will be denoted by $\boldsymbol{\varepsilon}^{\mathrm{THE}}$ and $\boldsymbol{\sigma}^{\mathrm{THE}}$ in Section 3 for clarity, but this distinction is not essential here. Notice that the 
average stress and strain are related by $\boldsymbol{\varepsilon}_{s}=\boldsymbol{G}_{s}\left(\boldsymbol{\sigma}_{s}\right)$ in each phase $s$, which, for typical nonlinear elastic behaviour, leads to overestimating the local, and therefore the overall stiffness. Formally, Eq. (3) are unchanged but they now only use per phase average linear localisation tensors $\boldsymbol{A}_{s}$ or $\boldsymbol{B}_{s}$, e.g.,

$$
\begin{aligned}
& \overline{\boldsymbol{\varepsilon}}=\tilde{\boldsymbol{M}}: \overline{\boldsymbol{\sigma}}+\tilde{\boldsymbol{\eta}}, \\
& \tilde{\boldsymbol{M}}=\sum_{s} c_{s} \boldsymbol{M}_{s}: \boldsymbol{B}_{s}, \\
& \tilde{\boldsymbol{\eta}}=\sum_{s} c_{s} \boldsymbol{\eta}_{s}: \boldsymbol{B}_{s}, \\
& \boldsymbol{B}_{s}=\langle\boldsymbol{B}\rangle_{s},
\end{aligned}
$$

with $c_{s}$ denoting the volume fraction of phase $s$ and $\langle\cdot\rangle$ the spatial average over phase $s$. Additional localisation equations, relating the reference stresses $\boldsymbol{\sigma}_{s}$, identified with the per phase stress averages in the thermoelastic comparison composite, to the global stress $\overline{\boldsymbol{\sigma}}$ and the prestrains $\boldsymbol{\eta}_{s}$ are still needed: they have to be specified independently, according to the chosen homogenisation scheme. Two-phase materials provide a noticeable exception since Levin's theorem (Levin, 1967) gives then directly

$$
c_{1}\left(\boldsymbol{\sigma}_{1}-\boldsymbol{B}_{1}: \overline{\boldsymbol{\sigma}}_{1}\right)=-c_{2}\left(\boldsymbol{\sigma}_{2}-\boldsymbol{B}_{2}: \overline{\boldsymbol{\sigma}}\right)=\left(\boldsymbol{M}_{1}-\boldsymbol{M}_{2}\right)^{-1}:(\tilde{\boldsymbol{\eta}}-\langle\boldsymbol{\eta}\rangle) .
$$

\subsection{Hashin-Shtrikman estimates}

For multiphase materials, we can adopt Hashin-Shtrikman type estimates for which the localisation equations read

$$
\boldsymbol{\sigma}_{s}=\left(\boldsymbol{I}+\boldsymbol{Q}^{0}: \boldsymbol{\delta} \boldsymbol{M}_{s}^{0}\right)^{-1}:\left[\overline{\boldsymbol{\sigma}}_{0}+\boldsymbol{Q}^{0}:\left(\tilde{\boldsymbol{\eta}}-\boldsymbol{\eta}_{s}\right)\right]
$$

with $\boldsymbol{I}$ is the-fourth order unit tensor, $\boldsymbol{\delta} \boldsymbol{M}_{s}^{0}$ the deviations of the local compliances with respect to those of the reference medium (say $\boldsymbol{M}^{0}$ ), $\overline{\boldsymbol{\sigma}}_{0}$ an auxiliary stress tensor ensuring $\langle\boldsymbol{\sigma}\rangle=\overline{\boldsymbol{\sigma}}$ and $\boldsymbol{Q}^{0}$ the stress Green tensor defined as

$$
Q^{0}=\int_{\mathscr{E}} \Delta^{0}\left(x-x^{\prime}\right) \mathrm{d} x^{\prime}, \quad x \in \mathscr{E} .
$$

Here $\boldsymbol{\Delta}^{0}$ is the stress Green operator of the reference medium and $\mathscr{E}$ is the representative ellipsoid associated with the assumed ellipsoidal spatial distribution (Willis, 1977) of the phases. Dual variants of Eqs. (7) and (8) are derived for $\boldsymbol{\varepsilon}_{s}$ and the strain Green tensor $\boldsymbol{P}^{0}$ upon substitution of $\boldsymbol{M}_{s}, \boldsymbol{M}^{0}, \overline{\boldsymbol{\sigma}}_{0}, \overline{\boldsymbol{\sigma}}, \tilde{\boldsymbol{\eta}}, \boldsymbol{\eta}_{s}$ and $\boldsymbol{\Delta}^{0}$ by $\boldsymbol{L}_{s}, \boldsymbol{L}^{0}, \overline{\boldsymbol{\varepsilon}}_{0}, \overline{\boldsymbol{\varepsilon}}, \tilde{\boldsymbol{\tau}}, \boldsymbol{\tau}_{s}$ and the strain Green operator $\boldsymbol{\Gamma}^{0}$, respectively. 
Hill's compliance constraint tensor $\boldsymbol{M}^{* 0}$ can be used to rewrite Eq. (7) as an interaction equation of an inclusion of phase $s$ embedded in an infinite reference medium:

$$
\boldsymbol{\varepsilon}_{s}-\overline{\boldsymbol{\varepsilon}}_{0}=-\boldsymbol{M}^{* 0}:\left(\boldsymbol{\sigma}_{s}-\overline{\boldsymbol{\sigma}}_{0}\right), \quad \boldsymbol{M}^{* 0}=\left(\boldsymbol{Q}^{0}\right)^{-1}-\boldsymbol{M}^{0}
$$

with $\boldsymbol{\varepsilon}_{s}=\boldsymbol{M}_{s}: \boldsymbol{\sigma}_{s}+\boldsymbol{\eta}_{s}$ and $\overline{\boldsymbol{\varepsilon}}_{0}=\boldsymbol{M}^{0}: \overline{\boldsymbol{\sigma}}_{0}+\tilde{\boldsymbol{\eta}}$ (the dual form would involve the stiffness constraint tensor $\left.\boldsymbol{L}^{* 0}=\left(\boldsymbol{M}^{* 0}\right)^{-1}=\left(\boldsymbol{P}^{0}\right)^{-1}-\boldsymbol{L}^{0}\right)$.

When the self-consistent scheme is used, the reference medium is the searched homogeneous equivalent medium: exponents 0 for $\boldsymbol{P}, \boldsymbol{Q}, \boldsymbol{\delta} \boldsymbol{L}_{s}$ and $\boldsymbol{\delta} \boldsymbol{M}_{s}$ must be changed to SC and $\boldsymbol{L}^{0}, \boldsymbol{M}^{0}$ into $\tilde{\boldsymbol{L}}^{S C}$ and $\tilde{\boldsymbol{M}}^{S C}$. Note that $\overline{\boldsymbol{\sigma}}_{0}$ (respectively $\overline{\boldsymbol{\varepsilon}}_{0}$ ) is then equal to $\overline{\boldsymbol{\sigma}}$ (respectively $\overline{\boldsymbol{\varepsilon}}$ ) and $\boldsymbol{B}_{S}^{\mathrm{SC}}$ and $\boldsymbol{M}^{* \mathrm{SC}}$ are linked by the relation:

$$
\boldsymbol{B}_{s}^{\mathrm{SC}}=\left(\boldsymbol{M}_{s}+\boldsymbol{M}^{* \mathrm{SC}}\right)^{-1}:\left(\tilde{\boldsymbol{M}}^{\mathrm{SC}}+\boldsymbol{M}^{* \mathrm{SC}}\right) .
$$

The affine formulation can be applied to these estimates by solving the implicit set of nonlinear equations (4), (5) and (7) for the unknown quantities $\boldsymbol{\sigma}_{s}$ (or from their dual expressions for $\boldsymbol{\varepsilon}_{s}$ ). This can be performed numerically by the use of a (modified) fixed-point iterative procedure: for a given macroscopic stress $\overline{\boldsymbol{\sigma}}$, we start at step $(k)$ with initial values $\boldsymbol{\sigma}_{s}^{(k)}$ obtained at step $(k-1)$ (for $k=1$, we set $\left.\boldsymbol{\sigma}_{s}^{(1)}=\overline{\boldsymbol{\sigma}}, \forall s\right)$, from which we derive $\boldsymbol{M}_{s}^{(k)}$ and $\boldsymbol{\eta}_{s}^{(k)}$ by Eq. (4) and $\tilde{\boldsymbol{\eta}}^{(k)}$ and $\tilde{\boldsymbol{M}}^{(k)}$ by Eq. (5). Then Eq. (7) gives $\boldsymbol{\sigma}_{s}^{(k+1)}$ as solution of the nonlinear equation

$$
\boldsymbol{\sigma}_{s}^{(k+1)}=\left(\boldsymbol{I}+\boldsymbol{Q}^{0(k)}: \boldsymbol{\delta} \boldsymbol{M}_{s}^{0(k)}\right)^{-1}:\left\{\overline{\boldsymbol{\sigma}}_{0}^{(k)}+\boldsymbol{Q}^{0(k)}:\left[\tilde{\boldsymbol{\eta}}^{(k)}-\boldsymbol{G}_{s}\left(\boldsymbol{\sigma}_{s}^{(k+1)}\right)+\boldsymbol{M}_{s}^{(k)}: \boldsymbol{\sigma}_{s}^{(k+1)}\right]\right\}
$$

where $\boldsymbol{\sigma}_{s}^{(k+1)}$ has been used instead of $\boldsymbol{\sigma}_{s}^{(k)}$ in the last two terms for accelerated convergence (this refers to a 'nonlinear inclusion' embedded in the infinite matrix defined by $\boldsymbol{M}^{0}$ and $\tilde{\boldsymbol{\eta}}^{(k)}$ subjected to $\overline{\boldsymbol{\sigma}}_{0}^{(k)}$ at infinity). The procedure is repeated until convergence is attained for the $\boldsymbol{\sigma}_{s}$. For the self-consistent scheme, the computation of $\tilde{\boldsymbol{M}}^{\mathrm{SC}}$ requires an additional iterative loop within each of the above steps.

\subsection{Comparison of the affine and tangent formulations}

The affine formulation makes use of the same linearisation scheme of the local constitutive equations as the one which was originally proposed for the selfconsistent modelling of power-law creep of polycrystals, within a finite strain framework, by Molinari et al. (1987) and then extended somewhat by Lebensohn and Tomé (1993). In this section, we first emphasise the main differences between this formulation, restricted to small strains, hereafter referred to as the 'tangent formulation' for reasons given in the sequel, and the affine formulation. An illustrative example is then used to compare the predictions of the affine, the tangent and the incremental formulations. Throughout this section $\boldsymbol{\varepsilon}$ will refer to the strain rate. 


\subsubsection{Analysis of the tangent formulation}

The affine and tangent formulations differ from each other in the way they tackle the localisation problem. The overall linearised constitutive equations have the same form (5) but the tangent effective compliances $\tilde{\boldsymbol{M}}^{\text {TAN }}$ and prestrain $\tilde{\boldsymbol{\eta}}^{\text {TAN }}$ obey

$$
\tilde{\boldsymbol{M}}^{\mathrm{TAN}}=\frac{\mathrm{d} \tilde{\boldsymbol{G}}^{\mathrm{TAN}}}{\mathrm{d} \overline{\boldsymbol{\sigma}}}(\overline{\boldsymbol{\sigma}}), \quad \tilde{\boldsymbol{\eta}}^{\mathrm{TAN}}=\tilde{\boldsymbol{G}}^{\mathrm{TAN}}(\overline{\boldsymbol{\sigma}})-\tilde{\boldsymbol{M}}^{\mathrm{TAN}}: \overline{\boldsymbol{\sigma}},
$$

instead of Eqs. (5b) and (5c). Here $\tilde{\boldsymbol{G}}^{\mathrm{TAN}}(\overline{\boldsymbol{\sigma}})$ is a 'tangent' estimate for the overall strain response. Such a treatment requires the stress dependence of this function to be known a priori. That is why the tangent formulation has been restricted to power-law creep with the same exponent $n$ throughout the material, a case for which the authors have argued, after Hutchinson $(1976)$, that $\tilde{\boldsymbol{G}}^{\mathrm{TAN}}(\overline{\boldsymbol{\sigma}})$ obeys the same power-law dependence. Let us adopt the same restriction for comparison; the local strain $\boldsymbol{\varepsilon}_{s}$ and prestrain $\boldsymbol{\eta}_{s}$ in each phase $s$ then satisfy:

$$
\begin{aligned}
& \boldsymbol{\varepsilon}_{s}=m \boldsymbol{M}_{s}: \boldsymbol{\sigma}_{s}, \\
& \boldsymbol{\eta}_{s}=(m-1) \boldsymbol{M}_{s}: \boldsymbol{\sigma}_{s}, \quad 0<m=\frac{1}{n}<1 .
\end{aligned}
$$

The same relations hold at the macroscopic level for the tangent approach:

$$
\begin{aligned}
& \overline{\boldsymbol{\varepsilon}}=m \tilde{\boldsymbol{M}}^{\mathrm{TAN}}: \overline{\boldsymbol{\sigma}}_{s}, \\
& \tilde{\boldsymbol{\eta}}^{\mathrm{TAN}}=(m-1) \tilde{\boldsymbol{M}}^{\mathrm{TAN}}: \overline{\boldsymbol{\sigma}} .
\end{aligned}
$$

Let us first show that the affine and tangent procedures do not coincide by proving that Eq. (14b) is not obeyed by $\tilde{\boldsymbol{\eta}}^{\mathrm{AFF}}$ and $\tilde{\boldsymbol{M}}^{\mathrm{AFF}}$. From Eqs. (5c) and (13b), we have

$$
\tilde{\boldsymbol{\eta}}^{\mathrm{AFF}}=\sum_{s} c_{s}{ }^{\mathrm{t}} \boldsymbol{B}_{s}: \boldsymbol{\eta}_{s}=(m-1) \sum_{s} c_{s}{ }^{\mathrm{t}} \boldsymbol{B}_{s}: \boldsymbol{M}_{s}: \boldsymbol{\sigma}_{s}
$$

where ${ }^{\mathrm{t}} \boldsymbol{B}$ is transposed from $\boldsymbol{B}$. From the classical thermoelastic relation between the macroscopic and local elastic strains and ${ }^{\mathrm{t}} \boldsymbol{B}$, for their affine analogues we have:

$$
\left.\tilde{\boldsymbol{M}}^{\mathrm{AFF}}: \overline{\boldsymbol{\sigma}}=\left\langle{ }^{\mathrm{t}} \boldsymbol{B}: \boldsymbol{M}: \boldsymbol{\sigma}\right\rangle=\sum_{s} c_{s}{ }^{\mathrm{t}} \boldsymbol{B}: \boldsymbol{M}_{s}: \boldsymbol{\sigma}\right\rangle_{s} .
$$

Since, due to the intraphase heterogeneity, we have in general

$$
\left\langle{ }^{\mathrm{t}} \boldsymbol{B}: \boldsymbol{M}_{s}: \boldsymbol{\sigma}\right\rangle_{s} \neq{ }^{\mathrm{t}} \boldsymbol{B}_{s}: \boldsymbol{M}_{s}: \boldsymbol{\sigma}_{s}, \quad \forall s,
$$

we conclude from Eqs. (15) and (17) that $\tilde{\boldsymbol{\eta}}^{\mathrm{AFF}} \neq(m-1) \tilde{\boldsymbol{M}}^{\mathrm{AFF}}: \overline{\boldsymbol{\sigma}}$, in contrast with 
Eq. (14). Thus, the affine formulation does not reduce to the tangent one which does not comply with the general framework of homogenised linear thermoelasticity.

An important difference can be pointed out between the tangent and affine treatments of the self-consistent scheme. Hill's expression (9), can be preferred to the more explicit form (7), but it has to be used simultaneously with the actual linearised constitutive equations which refer to a tangent description. On the other hand, according to the tangent approach, Hill's equation for phase $s$, applied to the self-consistent scheme, is combined with the relations

$$
\boldsymbol{\varepsilon}_{s}=m \boldsymbol{M}_{s}: \boldsymbol{\sigma}_{s} \quad \overline{\boldsymbol{\varepsilon}}=m \tilde{\boldsymbol{M}}^{\mathrm{TAN}}: \overline{\boldsymbol{\sigma}}
$$

which express a secant-like formulation. Consequently (see Lebensohn and Tomé, 1993), Eqs. (9) and (18) can be combined to give

$$
\boldsymbol{\sigma}_{s}=\boldsymbol{\beta}_{s}^{\mathrm{TAN}}: \overline{\boldsymbol{\sigma}}, \quad \boldsymbol{\beta}_{s}^{\mathrm{TAN}}=\left(m \boldsymbol{M}_{s}+\boldsymbol{M}^{* \mathrm{TAN}}\right)^{-1}:\left(m \boldsymbol{M}^{\mathrm{TAN}}+\boldsymbol{M}^{* \mathrm{TAN}}\right) .
$$

Referring to Eq. (10), this quasi-stress localisation tensor $\boldsymbol{\beta}_{s}^{\text {TAN }}$ combines a 'secant' description of the local and global behaviours with a tangent analysis of the inclusion/matrix interaction. This inconsistency is likely to be responsible for the odd behaviour of the tangent method at large $n$ values where its predictions tend towards the lower Reuss-type bound (see hereafter). The same comment can be made for the initial version of the tangent model (Molinari et al., 1987) too, which used an isotropic approximation of the overall compliance tensor $\tilde{\boldsymbol{M}}^{\mathrm{TAN}}$.

\subsubsection{Steady creep of fcc polycrystals}

The affine formulation is applied next to the self-consistent modelling of the steady creep of polycrystals for comparison with both the tangent and the incremental predictions. This polycrystal is an aggregate of perfectly bonded crystals which differ only by their lattice orientation. In each single crystal, the creep deformation is only due to glide on slip systems $\alpha$, with the unit normal to the slip plane $\boldsymbol{n}^{\alpha}$ and the unit slip direction $\boldsymbol{m}^{\alpha}$. The orientation tensor $\boldsymbol{R}^{\alpha}$ which relates the resolved shear stress to the stress tensor by $\tau^{\alpha}=\boldsymbol{R}^{\alpha}: \boldsymbol{\sigma}$ is

$$
\boldsymbol{R}^{\alpha}=\frac{1}{2}\left(\boldsymbol{n}^{\alpha} \otimes \boldsymbol{m}^{\alpha}+\boldsymbol{m}^{\alpha} \otimes \boldsymbol{n}^{\alpha}\right)
$$

where $\otimes$ denotes a tensorial product. Note that $\boldsymbol{m}^{\alpha}$ and $-\boldsymbol{m}^{\alpha}$ are taken to be associated with two different systems so that $\tau^{\alpha}$ is always nonnegative. Though the affine approach could be applied to any shear stress-shear strain rate relations, including dissimilar relations for different sets of slip systems, attention is restricted, for comparison with the tangent predictions, to power-law creep with the same exponent for all systems: $\gamma^{\alpha}=\gamma_{0}^{\alpha}\left(\tau^{\alpha} / \tau_{0}^{\alpha}\right)^{n}$ with $\gamma^{\alpha}$ the shear strain rate on system $\alpha, \gamma_{0}^{\alpha}$ a reference shear rate and $\tau_{0}^{\alpha}$ a (positive) reference shear stress. The relation between the local creep strain rate $\boldsymbol{\varepsilon}$ and stress $\boldsymbol{\sigma}$ in phase $s$, which refers to a set of grains with the same lattice orientation, reads 


$$
\left.\boldsymbol{\varepsilon}=\sum_{\alpha} \gamma^{\alpha}(\boldsymbol{\sigma}) \boldsymbol{R}^{\alpha}=\sum_{\alpha} \gamma_{0}^{\alpha} \frac{\boldsymbol{R}^{\alpha}: \boldsymbol{\sigma}}{\tau_{0}^{\alpha}}\right)^{n} \boldsymbol{R}^{\alpha}=\boldsymbol{G}_{s}(\sigma)
$$

and can be linearised about the reference stress $\sigma_{s}$ according to Eq. (4) with

$$
\left.\boldsymbol{M}_{s}=n \sum_{\alpha} \frac{\gamma_{0}^{\alpha}}{\tau_{0}^{\alpha}} \frac{\boldsymbol{R}^{\alpha}: \boldsymbol{\sigma}_{s}}{\tau_{0}^{\alpha}}\right)^{n-1} \boldsymbol{R}^{\alpha} \otimes \boldsymbol{R}^{\alpha}, \quad \boldsymbol{\eta}_{s}=(1-n) \boldsymbol{G}_{s}\left(\boldsymbol{\sigma}_{s}\right) .
$$

The linear self-consistent scheme with spherical symmetry is used to model an untextured fcc polycrystal. The affine estimate of the overall response $\overline{\boldsymbol{\varepsilon}}$ to a prescribed stress $\overline{\boldsymbol{\sigma}}$ is derived from Eqs. (5) and (22), combined with the localisation equations (7). Hill's constraint tensor $\boldsymbol{M}^{* \mathrm{SC}}$ can be used as well, which leads to estimates for $\boldsymbol{\sigma}_{s}$ through the resolution of the following set of equations:

$$
\begin{gathered}
\boldsymbol{\varepsilon}_{s}-\overline{\boldsymbol{\varepsilon}}=-\boldsymbol{M}^{* \mathrm{SC}}:\left(\boldsymbol{\sigma}_{s}-\overline{\boldsymbol{\sigma}}\right), \quad \boldsymbol{M}^{* \mathrm{SC}}=\left(\boldsymbol{Q}^{* \mathrm{SC}}\right)^{-1}-\tilde{\boldsymbol{M}}^{\mathrm{SC}} \\
\tilde{\boldsymbol{M}}^{\mathrm{SC}}=\sum_{s} c_{s} \boldsymbol{M}_{s}: \boldsymbol{B}_{s}^{\mathrm{SC}}, \quad \boldsymbol{B}_{s}^{\mathrm{SC}}=\left(\boldsymbol{I}+\boldsymbol{Q}^{\mathrm{SC}}: \boldsymbol{\delta} \boldsymbol{M}_{s}^{\mathrm{SC}}\right)^{-1} .
\end{gathered}
$$

These equations can also be used in order to derive both the incremental predictions, upon substitution of the tangent compliances $\boldsymbol{M}_{s}$ by the anisotropic secant ones $m \boldsymbol{M}_{s}$, and the tangent ones, upon substitution of the stress localisation tensors $\boldsymbol{B}_{s}^{\mathrm{SC}}$ by tensors $\boldsymbol{\beta}_{s}^{\text {TAN }}$ as given by Eq. (19). Thus the affine, tangent and incremental estimates can be determined with the same numerical procedure, adapted from the one proposed by Molinari et al. (1987).

Glide is assumed to occur on the twelve octahedral slip systems and shear strain rates to obey a power-law with homogeneous reference shear strain rates $\gamma_{0}$ and shear stresses $\tau_{0}$ throughout the polycrystal. For a tensile prescribed overall stress, the relation between the equivalent overall stress $\bar{\sigma}^{\text {eq }}$ and strain rate $\bar{\varepsilon}^{\mathrm{eq}}$ reads (Hutchinson, 1976) $\bar{\varepsilon}^{\mathrm{eq}}=\gamma_{0}\left(\bar{\sigma}^{\mathrm{eq}} / \tilde{\sigma}_{0}\right)^{n}$ so that the reference stress $\tilde{\sigma}_{0}$ governs the tensile overall creep response of the polycrystal. Due to the transverse isotropy of the creep compliance tensor and to incompressibility, $Q^{\mathrm{SC}}$ can be derived straightforwardly (Hutchinson, 1976). Classically, we take advantage of symmetries to reduce averages on one standard triangle and proceed with increasing values of $n$. Corresponding affine, tangent and incremental estimates of $\tilde{\sigma}_{0}$ as well as upper Voigt-Taylor and lower Reuss bounds are reported in Fig. 1. As pointed out by Hutchinson (1976), Hill's incremental estimate tends towards the Voigt-Taylor upper bound while, as expected, the affine prediction is significantly softer and is likely to tend towards some intermediate value between the Voigt and Reuss bounds (additional evidence for this will be found in Fig. 2 for two-phase materials). The tangent estimate softens as $n$ increases, which enhances the intracrystalline heterogeneity, and deviates from the affine one tending towards the Reuss bound. 


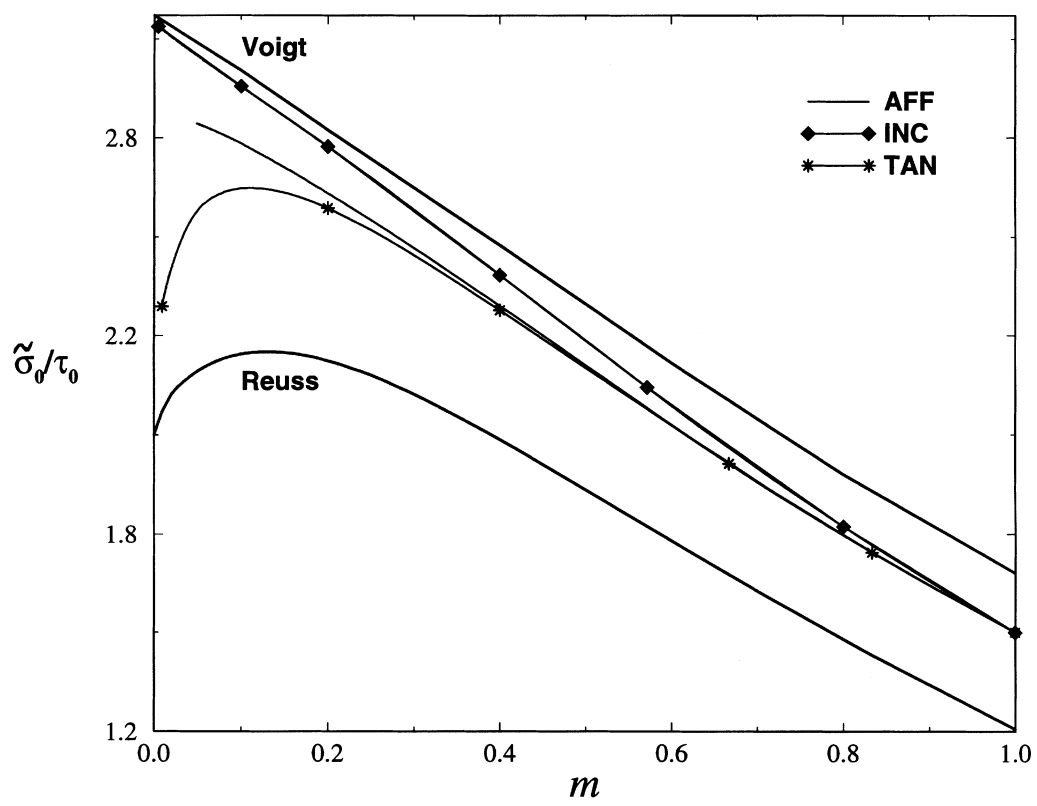

Fig. 1. Evolution of the normalised reference stress $\tilde{\sigma}_{0} / \tau_{0}$ of an untextured fcc polycrystal with the strain-rate sensitivity $m=1 / n$. Predictions of the affine (AFF), incremental (INC) and tangent (TAN) procedures combined with the self-consistent linear estimate, as well as Voigt and Reuss bounds are plotted for uniaxial tension.

\section{Limitations of the affine procedure}

\subsection{Effective potential and duality gap}

All previous derivations have been written in terms of stress-strain relations. For nonlinear elasticity or steady state creep (viscoplasticity), the local behaviour of the constitutive phases derives from a strain potential (or dissipation) $w_{s}(\boldsymbol{\varepsilon})$ or a stress potential $u_{s}(\boldsymbol{\sigma})$, which are Legendre transforms of each other:

$$
\boldsymbol{\sigma}=\frac{\mathrm{d} w_{s}}{\mathrm{~d} \boldsymbol{\varepsilon}}(\boldsymbol{\varepsilon}), \quad \boldsymbol{\varepsilon}=\frac{\mathrm{d} u_{s}}{\mathrm{~d} \boldsymbol{\sigma}}(\boldsymbol{\sigma}) \quad \text { and } \quad u_{s}(\boldsymbol{\sigma})=\sup _{\boldsymbol{\varepsilon}}\left[\boldsymbol{\varepsilon}: \boldsymbol{\sigma}-w_{s}(\boldsymbol{\varepsilon})\right] .
$$

The overall behaviour derives also from a strain $\tilde{w}(\overline{\boldsymbol{\varepsilon}})$ or a stress $\tilde{u}(\overline{\boldsymbol{\sigma}})$ potential, which are Legendre transforms of each other as well, and are given by:

$$
\tilde{w}(\overline{\boldsymbol{\varepsilon}})=\inf _{\boldsymbol{\varepsilon} \in \mathscr{K}(\overline{\boldsymbol{\varepsilon}})}\left\langle\sum_{S} \kappa_{S}(\boldsymbol{x}) w_{S}(\boldsymbol{\varepsilon})\right\rangle \quad \text { and } \quad \tilde{u}(\overline{\boldsymbol{\sigma}})=\inf _{\sigma \in \mathscr{S}(\overline{\boldsymbol{\sigma}})}\left\langle\sum_{S} \kappa_{S}(\boldsymbol{x}) u_{S}(\boldsymbol{\sigma})\right\rangle,
$$

where $\mathscr{K}(\overline{\boldsymbol{\varepsilon}})$ (or $\mathscr{S}(\overline{\boldsymbol{\sigma}})$ ) is the set of compatible strain (or statically admissible stress) fields with average $\overline{\boldsymbol{\varepsilon}}$ (or $\overline{\boldsymbol{\sigma}}$ ); $\kappa_{s}$ is the characteristic function of phase $s$. 
The question of the existence of such effective potentials from which the stressstrain relation predicted by the affine procedure would derive can be appropriately addressed through the comparison between the affine procedure and the secondorder procedure recently proposed by Ponte Castañeda (1996), which, by construction, derives from a potential. A related question is the equivalence of the prescribed strain approach and the dual approach of a prescribed overall stress.

\subsubsection{The second-order procedure}

We provide here a simplified description of this procedure and refer to Ponte Castañeda (1996) and Ponte Castañeda and Suquet (1998) for more details. It is based on a second-order Taylor expansion of the strain potential in each phase $s$ about some reference strain $\boldsymbol{\varepsilon}_{s}$, to be specified later:

$$
w_{s}(\boldsymbol{\varepsilon}) \approx w_{s}\left(\boldsymbol{\varepsilon}_{s}\right)+\boldsymbol{\sigma}_{s}:\left(\boldsymbol{\varepsilon}-\boldsymbol{\varepsilon}_{s}\right)+\frac{1}{2}\left(\boldsymbol{\varepsilon}-\boldsymbol{\varepsilon}_{s}\right): \boldsymbol{L}_{s}:\left(\boldsymbol{\varepsilon}-\boldsymbol{\varepsilon}_{s}\right)
$$

where $\boldsymbol{\sigma}_{s}=\frac{\mathrm{d} w_{s}}{\mathrm{~d} \boldsymbol{\varepsilon}}\left(\boldsymbol{\varepsilon}_{S}\right)$ is the stress associated to the reference strain and $\boldsymbol{L}_{s}=\frac{\mathrm{d}^{2} w_{s}}{\mathrm{~d} \boldsymbol{\varepsilon} \boldsymbol{\varepsilon}}\left(\boldsymbol{\varepsilon}_{s}\right)$ is the tangent modulus at the same reference strain. Such an expansion for the local potential is equivalent to a first-order expansion similar to Eq. (1) for the stressstrain relation, when $\boldsymbol{H}_{s}=\frac{\mathrm{d} w_{s}}{\mathrm{~d} \varepsilon}$. Combining Eqs. (25) and (26) provides an estimate $\tilde{w}^{\mathrm{SOE}}$ for the effective potential $\tilde{w}(\overline{\boldsymbol{\varepsilon}})$ of the composite:

$$
\tilde{w}^{\mathrm{SOE}}\left(\overline{\boldsymbol{\varepsilon}}, \boldsymbol{\varepsilon}_{1}, \ldots, \boldsymbol{\varepsilon}_{N}\right)=\sum_{s} c_{s}\left(w_{s}\left(\boldsymbol{\varepsilon}_{s}\right)-\boldsymbol{\sigma}_{s}: \boldsymbol{\varepsilon}_{s}+\frac{1}{2} \boldsymbol{\varepsilon}_{s}: \boldsymbol{L}_{s}: \boldsymbol{\varepsilon}_{s}\right)+\tilde{P}\left(\overline{\boldsymbol{\varepsilon}}, \boldsymbol{\varepsilon}_{1}, \ldots, \boldsymbol{\varepsilon}_{N}\right)
$$

with

$$
\left.\left.\tilde{P}\left(\overline{\boldsymbol{\varepsilon}}, \boldsymbol{\varepsilon}_{1}, \ldots, \boldsymbol{\varepsilon}_{N}\right)=\inf _{\boldsymbol{\varepsilon} \in \mathscr{K}(\overline{\boldsymbol{\varepsilon}})}\left\langle\frac{1}{2} \boldsymbol{\varepsilon}: \sum_{s} \kappa_{s} \boldsymbol{L}_{s}\right): \boldsymbol{\varepsilon}+\sum_{s} \kappa_{s} \boldsymbol{\tau}_{s}\right): \boldsymbol{\varepsilon}\right\rangle,
$$

where, as in Eq. (1), $\boldsymbol{\tau}_{s}=\boldsymbol{\sigma}_{s}-\boldsymbol{L}_{s}: \boldsymbol{\varepsilon}_{s}$. The last minimisation is a problem for a linear thermoelastic comparison composite with the phase distribution of the nonlinear composite and with homogeneous local moduli $\boldsymbol{L}_{s}$ and prestresses $\boldsymbol{\tau}_{s}$ in each phase s. Its solution takes the form (Willis, 1981):

$$
\tilde{P}(\overline{\boldsymbol{\varepsilon}})=\frac{1}{2} \overline{\boldsymbol{\varepsilon}}: \tilde{\boldsymbol{L}}: \overline{\boldsymbol{\varepsilon}}+\tilde{\tau}: \overline{\boldsymbol{\varepsilon}}+\tilde{f},
$$

where the effective moduli $\tilde{\boldsymbol{L}}$ and the effective prestress $\tilde{\boldsymbol{\tau}}$ are computed according to the dual expression of Eq. (5) with help of some linear localisation tensors $\boldsymbol{A}_{s}$ relevant to the microstructure $(\tilde{f}$ is the elastic energy at vanishing overall strain, related to internal strain incompatibilities). The macroscopic stress is computed by taking the derivative of $\tilde{w}(\overline{\boldsymbol{\varepsilon}})$ with respect to $\overline{\boldsymbol{\varepsilon}}$. One can check that

$$
\frac{\partial \tilde{P}}{\partial \boldsymbol{\varepsilon}_{s}}=\frac{1}{2} c_{s}\left\langle\boldsymbol{\varepsilon}: \boldsymbol{N}_{s}: \boldsymbol{\varepsilon}\right\rangle_{s}-c_{s}\langle\boldsymbol{\varepsilon}\rangle_{s}: \boldsymbol{N}_{s}: \boldsymbol{\varepsilon}_{s} \text { with } \boldsymbol{N}_{s}=\frac{\mathrm{d}^{3} w_{s}}{\mathrm{~d} \boldsymbol{\varepsilon} \mathrm{d} \boldsymbol{\varepsilon} \mathrm{d} \boldsymbol{\varepsilon}}\left(\boldsymbol{\varepsilon}_{s}\right),
$$


so that

$$
\overline{\boldsymbol{\sigma}}^{\mathrm{SOE}}=\overline{\boldsymbol{\sigma}}^{\mathrm{THE}}+\frac{1}{2} \sum_{s} c_{s}\left[\boldsymbol{N}_{s}: \frac{\mathrm{d} \boldsymbol{\varepsilon}_{s}}{\mathrm{~d} \overline{\boldsymbol{\varepsilon}}}\right]::\left(\boldsymbol{\varepsilon}-\boldsymbol{\varepsilon}_{s}\right) \otimes\left(\boldsymbol{\varepsilon}-\boldsymbol{\varepsilon}_{s}\right)
$$

where $\overline{\boldsymbol{\sigma}}^{\mathrm{THE}}=\tilde{\boldsymbol{L}}: \overline{\boldsymbol{\varepsilon}}+\tilde{\boldsymbol{\tau}}$ is the macroscopic stress in the thermoelastic comparison composite. The above expressions provide an estimate for the effective strain potential and the effective stress related to the macroscopic strain $\bar{\varepsilon}$ for any choice of reference strains $\boldsymbol{\varepsilon}_{s}$. An appropriate choice is to prescribe, as in Section 2.2, the reference strains to be equal to the average strains in every phase of the linear thermoelastic comparison composite subjected to the same overall strain: $\forall s, \boldsymbol{\varepsilon}_{s}=$ $\langle\boldsymbol{\varepsilon}\rangle_{s}$. This prescription leads to a simpler expression of the estimate of the overall nonlinear potential (Ponte Castañeda and Suquet, 1998):

$$
\tilde{w}^{\mathrm{SOE}}(\overline{\boldsymbol{\varepsilon}})=\sum_{s} c_{s}\left[w_{s}\left(\boldsymbol{\varepsilon}_{s}\right)+\frac{1}{2} \boldsymbol{\sigma}_{s}\left(\boldsymbol{\varepsilon}_{s}\right):\left(\overline{\boldsymbol{\varepsilon}}-\boldsymbol{\varepsilon}_{s}\right)\right] .
$$

The second-order procedure can also be applied in the dual situation of a prescribed stress and leads to estimates for the effective stress potential and the corresponding macroscopic strain. Second-order Taylor expansions of the local stress potential about some reference stresses $\boldsymbol{\sigma}_{s}^{\sigma}$, when plugged into Eq. (25), provide an estimate for the effective stress potential involving the stress potential of some linear thermoelastic comparison composite:

$$
\tilde{u}^{\mathrm{SOE}}(\overline{\boldsymbol{\sigma}})=\sum_{s} c_{s}\left(u_{s}\left(\boldsymbol{\sigma}_{s}^{\sigma}\right)-\boldsymbol{\varepsilon}_{s}^{\sigma}: \boldsymbol{\sigma}_{s}^{\sigma}+\frac{1}{2} \boldsymbol{\sigma}_{s}^{\sigma}: \boldsymbol{M}_{s}: \boldsymbol{\sigma}_{s}^{\sigma}\right)+\frac{1}{2} \overline{\boldsymbol{\sigma}}: \tilde{\boldsymbol{M}}: \overline{\boldsymbol{\sigma}}+\tilde{\boldsymbol{\eta}}: \overline{\boldsymbol{\sigma}}+\tilde{g}
$$

where, as previously, $\boldsymbol{\varepsilon}_{s}^{\sigma}=\frac{\mathrm{d} u_{s}}{\mathrm{~d} \boldsymbol{\sigma}}\left(\boldsymbol{\sigma}_{s}^{\sigma}\right)$ and $\boldsymbol{M}_{s}=\frac{\mathrm{d}^{2} u_{s}}{\mathrm{~d} \boldsymbol{\sigma} \mathrm{d} \boldsymbol{\sigma}}\left(\boldsymbol{\sigma}_{s}^{\sigma}\right), \boldsymbol{\eta}_{s}=\boldsymbol{\varepsilon}_{s}^{\sigma}-\boldsymbol{M}_{s}: \boldsymbol{\sigma}_{s}^{\sigma}$ and $\tilde{\boldsymbol{M}}$ and $\tilde{\boldsymbol{\eta}}$ are the effective compliances and prestrains of this comparison composite ( $\tilde{g}$ is its complementary energy at vanishing overall stress, related to residual stresses). The overall strain is obtained by derivation with respect to $\overline{\boldsymbol{\sigma}}$ :

$$
\left.\overline{\boldsymbol{\varepsilon}}^{\mathrm{SOE}}=\overline{\boldsymbol{\varepsilon}}^{\mathrm{THE}}+\frac{1}{2} \sum_{s} c_{s}\left[\boldsymbol{O}_{s}: \frac{\mathrm{d} \boldsymbol{\sigma}_{s}^{\sigma}}{\mathrm{d} \overline{\boldsymbol{\sigma}}}\right]::\left(\boldsymbol{\sigma}-\boldsymbol{\sigma}_{s}^{\sigma}\right) \quad\left(\boldsymbol{\sigma}-\boldsymbol{\sigma}_{s}^{\sigma}\right)\right\rangle_{s}
$$

where $\overline{\boldsymbol{\varepsilon}}^{\mathrm{THE}}=\tilde{\boldsymbol{M}}: \overline{\boldsymbol{\sigma}}+\tilde{\boldsymbol{\eta}}$ is the macroscopic stress in the thermoelastic comparison composite and with $\boldsymbol{O}_{s}=\frac{\mathrm{d}^{3} u_{s}}{\mathrm{~d} \boldsymbol{d} \mathrm{d} \boldsymbol{d} \boldsymbol{\sigma}}\left(\boldsymbol{\sigma}_{s}^{\sigma}\right)$. The reference stresses are the average stresses in the phases in the thermoelastic comparison composite: $\boldsymbol{\sigma}_{s}^{\sigma}=\left\langle\boldsymbol{\sigma}^{\sigma}\right\rangle_{s}$.

\subsubsection{Comparison between affine and second-order procedures}

The linear thermoelastic comparison composite referred to by the affine and the second-order procedure for a given prescribed strain $\overline{\boldsymbol{\varepsilon}}$ are identical. Both approaches differ only by the way the overall stress is computed. While in the affine procedure it is identified to the overall stress in the comparison composite according to $\overline{\boldsymbol{\sigma}}^{\mathrm{AFF}}=\overline{\boldsymbol{\sigma}}^{\mathrm{THE}}$, it is given by Eq. (31) in the second-order procedure. Both results are different because of the additional term in Eq. (31) which reads, 
with $\boldsymbol{\varepsilon}_{s}=\langle\boldsymbol{\varepsilon}\rangle_{s}$ :

$$
\overline{\boldsymbol{\sigma}}^{\mathrm{SOE}}-\overline{\boldsymbol{\sigma}}^{\mathrm{AFF}}=\frac{1}{2} \sum_{s} c_{s}\left[\boldsymbol{N}_{s}: \frac{\mathrm{d} \boldsymbol{\varepsilon}_{s}}{\mathrm{~d} \overline{\boldsymbol{\varepsilon}}}\right]::\left[\langle\boldsymbol{\varepsilon} \otimes \boldsymbol{\varepsilon}\rangle_{s}-\langle\boldsymbol{\varepsilon}\rangle_{s} \otimes\langle\boldsymbol{\varepsilon}\rangle_{s}\right] .
$$

The second moment $\langle\boldsymbol{\varepsilon} \otimes \boldsymbol{\varepsilon}\rangle_{S}$ of local strains is generally larger than $\langle\boldsymbol{\varepsilon}\rangle_{s} \otimes\langle\boldsymbol{\varepsilon}\rangle_{S}$ (in the sense of quadratic forms) because of the strain heterogeneity within the phases in the comparison composite. Exceptions correspond to materials with a phase distribution such that the local fields in the comparison composite are uniform in all phases exhibiting a nonlinear behaviour (i.e., $\boldsymbol{N}_{s} \neq 0$ ): examples of such materials are laminates and matrix-inclusion composites with a linear matrix and nonlinear ellipsoidal inclusions at low volume fraction. This intraphase heterogeneity is also the reason for which the affine estimate does generally not derive from an effective potential. Note also that, because of the additional term in Eq. (31), it does not make sense, for the second-order procedure, to identify the local stresses in the comparison composite to the local stresses in the nonlinear composite.

The question of the existence or not of a duality gap can be addressed in the following way: if $\overline{\boldsymbol{\sigma}}(\overline{\boldsymbol{\varepsilon}})$ is the macroscopic stress due to the prescribed strain $\overline{\boldsymbol{\varepsilon}}$ according to some scheme, does the strain due to this stress according to the dual version of the same scheme coincide with $\overline{\boldsymbol{\varepsilon}}$ ? The answer is yes for the affine procedure. This can be made clear by noting that the stresses $\boldsymbol{\sigma}_{s}=\frac{\mathrm{d} w_{s}}{\mathrm{~d} \boldsymbol{\varepsilon}}\left(\boldsymbol{\varepsilon}_{s}\right)$ related to the reference strains which solve the equations governing the affine procedure for the prescribed strain $\overline{\boldsymbol{\varepsilon}}$ solve the equations that characterise the reference stresses in the dual approach for a prescribed stress $\overline{\boldsymbol{\sigma}}^{\mathrm{AFF}}(\overline{\boldsymbol{\varepsilon}})=\overline{\boldsymbol{\sigma}}^{\mathrm{THE}}(\overline{\boldsymbol{\varepsilon}})$. This is mostly because $\boldsymbol{L}_{s}\left(\boldsymbol{\varepsilon}_{s}\right)$ and $\boldsymbol{M}_{s}\left(\boldsymbol{\sigma}_{s}\right)$ are inverse of each other. But these stresses $\boldsymbol{\sigma}_{s}$ in general do not solve the corresponding equations for an overall stress $\overline{\boldsymbol{\sigma}}^{\mathrm{SOE}}(\overline{\boldsymbol{\varepsilon}})$. The reference stresses in the dual second-order approach for a prescribed stress $\overline{\boldsymbol{\sigma}}^{\mathrm{SOE}}(\overline{\boldsymbol{\varepsilon}})$ are thus different from $\boldsymbol{\sigma}_{s}$ and that is why they were referred to in the previous section using the exponent $\sigma$ : there are two different second-order estimates, the first one, based on the strain potential expansion, being generally more appropriate than the second one for the type of nonlinearities exhibited by ductile composites.

At this stage it is worth mentioning that there might be some other nonstandard, more appropriate choices of reference strains or stresses, for both the affine and the second-order procedure. In particular, the prescription

$$
\forall s \quad\left\langle\left(\boldsymbol{\varepsilon}-\boldsymbol{\varepsilon}_{s}\right): \boldsymbol{N}_{s}:\left(\boldsymbol{\varepsilon}-\boldsymbol{\varepsilon}_{s}\right)_{s}=0\right.
$$

ensuring that $\overline{\boldsymbol{\sigma}}^{\mathrm{SOE}}=\overline{\boldsymbol{\sigma}}^{\mathrm{AFF}}=\overline{\boldsymbol{\sigma}}^{\mathrm{THE}}$, would guarantee the existence of an overall potential for the affine procedure and would suppress the duality gap in the second order one. Unfortunately, such a definition leads to a dead end in the case of power-law type materials, such that $w_{s}(\lambda \boldsymbol{\varepsilon})=\lambda^{m+1} w_{s}(\boldsymbol{\varepsilon}), \forall \lambda>0$. For such materials the reference strains are homogeneous functions of degree one of the overall strain so that $\boldsymbol{L}_{s}(\overline{\boldsymbol{\varepsilon}})$ is homogeneous of degree $m-1$ and, by Euler's 
formula, $\boldsymbol{N}_{s}: \frac{\mathrm{d} \boldsymbol{\varepsilon}_{s}}{\mathrm{~d} \overline{\boldsymbol{\varepsilon}}}: \overline{\boldsymbol{\varepsilon}}=(m-1) \boldsymbol{L}_{s}(\overline{\boldsymbol{\varepsilon}})$. The general result (31) reads then

$$
2\left(\overline{\boldsymbol{\sigma}}^{\mathrm{SOE}}-\overline{\boldsymbol{\sigma}}^{\mathrm{AFF}}\right): \overline{\boldsymbol{\varepsilon}}=(m-1) \sum_{s}\left\langle\left(\boldsymbol{\varepsilon}-\boldsymbol{\varepsilon}_{s}\right): \boldsymbol{L}_{s}:\left(\boldsymbol{\varepsilon}-\boldsymbol{\varepsilon}_{s}\right)_{s}\right.
$$

which is negative because $\boldsymbol{L}_{s}$ is positive definite and $m \leq 1$. The dual result is:

$$
2\left(\overline{\boldsymbol{\varepsilon}}^{\mathrm{SOE}}-\overline{\boldsymbol{\varepsilon}}^{\mathrm{AFF}}\right): \overline{\boldsymbol{\sigma}}=(n-1) \sum_{s} c_{s}\left(\left(\boldsymbol{\sigma}-\boldsymbol{\sigma}_{s}\right): \boldsymbol{M}_{s}:\left(\boldsymbol{\sigma}-\boldsymbol{\sigma}_{s}^{\sigma}\right)\right\rangle_{s} \geq 0
$$

This proves that both second-order estimates are always weaker than the affine one for power-law type materials, regardless of the definition used for the reference strains or stresses; equality can only be achieved in materials exhibiting uniform local fields in the nonlinear phases, as those mentioned above.

\subsection{Weakly inhomogeneous composites}

\subsubsection{Exact second-order expansion}

The composites considered in this section have a small contrast. More specifically the energy $w_{s}$ of the phases differs from the energy $w_{0}$ of a homogeneous nonlinear reference medium by a small quantity measured by a small parameter $t: w_{s}(\boldsymbol{\varepsilon})=w_{0}(\boldsymbol{\varepsilon})+t \delta w_{s}(\boldsymbol{\varepsilon})$. The following notations related to the homogeneous reference medium and to the perturbation will be useful in the sequel

$$
\boldsymbol{L}_{0}=\frac{\mathrm{d}^{2} w_{0}}{\mathrm{~d} \boldsymbol{\varepsilon} \mathrm{d} \boldsymbol{\varepsilon}}(\overline{\boldsymbol{\varepsilon}}), \quad \boldsymbol{N}_{0}=\frac{\mathrm{d}^{3} w_{0}}{\mathrm{~d} \boldsymbol{\varepsilon} \mathrm{d} \boldsymbol{\varepsilon} \mathrm{d} \boldsymbol{\varepsilon}}(\overline{\boldsymbol{\varepsilon}}), \quad \boldsymbol{\tau}_{s}=\frac{\mathrm{d}}{\mathrm{d} \boldsymbol{\varepsilon}} \delta w_{s}(\overline{\boldsymbol{\varepsilon}}), \quad \boldsymbol{\delta} \boldsymbol{L}_{s}=\frac{\mathrm{d}^{2}}{\mathrm{~d} \boldsymbol{\varepsilon} \mathrm{d} \boldsymbol{\varepsilon}} \delta w_{s}(\overline{\boldsymbol{\varepsilon}}) .
$$

An asymptotic expansion with respect to the contrast for the exact fields $\boldsymbol{\varepsilon}$ and $\boldsymbol{\sigma}$ in the nonlinear composite and for the exact effective energy has been given by Suquet and Ponte Castañeda (1993). This expansion to second-order for the energy reads

$$
\tilde{w}(\overline{\boldsymbol{\varepsilon}})=w_{0}(\overline{\boldsymbol{\varepsilon}})+t\langle\delta w\rangle(\overline{\boldsymbol{\varepsilon}})-\frac{t^{2}}{2}\left\langle\dot{\boldsymbol{\varepsilon}}_{0}: \boldsymbol{L}_{0}: \dot{\boldsymbol{\varepsilon}}_{0}\right\rangle+O\left(t^{3}\right)
$$

where $\dot{\boldsymbol{\varepsilon}}_{0}$ is the solution of the following linear 'thermoelastic' problem

$$
\dot{\boldsymbol{\sigma}}_{0}(\boldsymbol{x})=\boldsymbol{L}_{0}: \dot{\boldsymbol{\varepsilon}}_{0}(\boldsymbol{x})+\sum_{s} \kappa_{s}(\boldsymbol{x}) \boldsymbol{\tau}_{s}, \quad \operatorname{div}\left(\dot{\boldsymbol{\sigma}}_{0}\right)=0, \quad\left\langle\dot{\boldsymbol{\varepsilon}}_{0}\right\rangle=0 .
$$

The expansion to second-order in the contrast of the exact effective stress-strain relation for the nonlinear composite derives from Eq. (40):

$$
\overline{\boldsymbol{\sigma}} \approx \frac{\mathrm{d} w_{0}}{\mathrm{~d} \boldsymbol{\varepsilon}}(\overline{\boldsymbol{\varepsilon}})+t \frac{\mathrm{d}}{\mathrm{d} \boldsymbol{\varepsilon}}\langle\delta w\rangle(\overline{\boldsymbol{\varepsilon}})-\frac{t^{2}}{2}\left[\left\langle\dot{\boldsymbol{\varepsilon}}_{0}: \boldsymbol{N}_{0}: \dot{\boldsymbol{\varepsilon}}_{0}\right\rangle+2\left\langle\dot{\boldsymbol{\varepsilon}}_{0}: \boldsymbol{L}_{0}: \frac{\mathrm{d} \dot{\boldsymbol{\varepsilon}}_{0}}{\mathrm{~d} \overline{\boldsymbol{\varepsilon}}}\right\rangle\right]
$$

and this expression can be simplified (using the relations obtained by 
differentiating Eq. (41) with respect to $\overline{\boldsymbol{\varepsilon}}$ ) into

$$
\overline{\boldsymbol{\sigma}} \approx \frac{\mathrm{d} w_{0}}{\mathrm{~d} \boldsymbol{\varepsilon}}(\overline{\boldsymbol{\varepsilon}})+t \frac{\mathrm{d}}{\mathrm{d} \boldsymbol{\varepsilon}}\langle\delta w\rangle(\overline{\boldsymbol{\varepsilon}})+\frac{t^{2}}{2}\left[\left\langle\dot{\boldsymbol{\varepsilon}}_{0}: \boldsymbol{N}_{0}: \dot{\boldsymbol{\varepsilon}}_{0}\right\rangle+2\left\langle\boldsymbol{\delta} \boldsymbol{L}: \dot{\boldsymbol{\varepsilon}}_{0}\right]+O\left(t^{3}\right) .\right.
$$

\subsubsection{The affine method for small contrast}

The predictions of the second-order and affine estimates, both for the local fields and for the effective constitutive law, can be expanded to second order in the contrast. Regarding the local fields, the Taylor expansion of the fields $\boldsymbol{\sigma}^{\mathrm{THE}}$ and $\boldsymbol{\varepsilon}^{\mathrm{THE}}$ in the linear thermoelastic comparison medium can be obtained for small contrast $t$ by considering the successive derivatives with respect to $t$ of the systems of equations in the thermoelastic comparison composite satisfied by $\boldsymbol{\sigma}^{\mathrm{THE}}$ and $\boldsymbol{\varepsilon}^{\mathrm{THE}}$ :

$$
\boldsymbol{\varepsilon}^{\mathrm{THE}}=\boldsymbol{\varepsilon}_{0}^{\mathrm{THE}}+t \dot{\boldsymbol{\varepsilon}}_{0}^{\mathrm{THE}}+\frac{t^{2}}{2} \ddot{\boldsymbol{\varepsilon}}_{0}^{\mathrm{THE}}+O\left(t^{3}\right) \quad\left(\text { same form for } \boldsymbol{\sigma}^{\mathrm{THE}}\right) .
$$

The terms of order 0 in these expansions are the homogeneous fields in the homogeneous nonlinear reference medium with energy $w_{0}: \boldsymbol{\varepsilon}_{0}^{\mathrm{THE}}=\overline{\boldsymbol{\varepsilon}}, \boldsymbol{\sigma}_{0}^{\mathrm{THE}}=\frac{\mathrm{d} w_{0}}{\mathrm{~d} \boldsymbol{\varepsilon}}(\overline{\boldsymbol{\varepsilon}})$. To order $1,\left(\dot{\boldsymbol{\varepsilon}}_{0}^{\mathrm{THE}}, \dot{\boldsymbol{\sigma}}_{0}^{\mathrm{THE}}\right)$ coincide with the solution of Eq. (41). The terms of order 2 are obtained by taking the second derivative with respect to $t$ of the system of equations satisfied by $\boldsymbol{\sigma}^{\mathrm{THE}}, \boldsymbol{\varepsilon}^{\mathrm{THE}}$ at $t=0$. It is found that the fields $\ddot{\boldsymbol{\varepsilon}}_{0}^{\mathrm{THE}}$ and $\ddot{\boldsymbol{\sigma}}_{0}^{\mathrm{THE}}$ do not coincide with the exact second-order terms in the expansion of the actual fields in the nonlinear composite. Therefore, the approximate fields $\boldsymbol{\sigma}^{\mathrm{THE}}, \boldsymbol{\varepsilon}^{\mathrm{THE}}$ coincide with the actual fields $\boldsymbol{\sigma}, \boldsymbol{\varepsilon}$ in the nonlinear composite up to order 1 in the contrast, but not to higher order in general.

Regarding the effective constitutive relation, the prediction of the second-order procedure for the energy (and therefore for the constitutive relations) is known (Ponte Castañeda, 1996) to be exact to second-order in the contrast. Therefore, it also coincides with Eq. (43). The expansion to second-order in the contrast of the prediction of the affine procedure can be performed either by taking the spatial average of the expansion (44) for $\boldsymbol{\sigma}^{\mathrm{THE}}$ to second-order or by using the expansion (44) for $\varepsilon^{\mathrm{THE}}$ to first-order and the relations (35) and (43). The final result reads

$$
\overline{\boldsymbol{\sigma}}^{\mathrm{AFF}}=\frac{\mathrm{d} w_{0}}{\mathrm{~d} \boldsymbol{\varepsilon}}(\overline{\boldsymbol{\varepsilon}})+t \frac{\mathrm{d}}{\mathrm{d} \boldsymbol{\varepsilon}}\langle\delta w\rangle(\overline{\boldsymbol{\varepsilon}})+\frac{t^{2}}{2}\left[\sum_{s} c_{s}\left\langle\dot{\boldsymbol{\varepsilon}}_{0}\right\rangle_{s}: \boldsymbol{N}_{0}:\left\langle\dot{\boldsymbol{\varepsilon}}_{0}\right\rangle_{s}+2\left\langle\boldsymbol{\delta} \boldsymbol{L}: \dot{\boldsymbol{\varepsilon}}_{0}\right]+O\left(t^{3}\right) .\right.
$$

The affine procedure is therefore not exact to second order in the contrast, except when the first order correction $\dot{\boldsymbol{\varepsilon}}_{0}$ of the strain field is constant in each phase.

\subsection{Application to porous materials}

Nonlinear porous materials should exhibit a nonlinear answer under pure hydrostatic load, even when the nonlinear matrix is incompressible. On the contrary, the affine estimate for such materials exhibits a linear dependence of the volume change on the hydrostatic pressure at a fixed, possibly non-vanishing, 
macroscopic deviatoric load. Such a limitation is in fact common to all nonlinear extensions that assume, explicitly or not, that the average stress of a given phase is related to the average strain by the local constitutive law of this phase:

$$
\langle\boldsymbol{\varepsilon}\rangle_{s}=\frac{\mathrm{d} u_{s}}{\mathrm{~d} \boldsymbol{\sigma}}\left(\langle\boldsymbol{\sigma}\rangle_{s}\right)
$$

and make use of some linearisation of the local constitutive law about the average strain or stress in each phase. This is the case for the classical incremental procedure, the classical secant schemes and the tangent model, in their anisotropic as well as isotropic variants, and the present affine procedure; but it is neither the case for the variational estimates (or the modified secant procedure) nor the second-order estimate.

The reason for this limitation can be made clear as follows. Consider a porous materials with a pore volume fraction $c$ subjected to the overall stress $\overline{\boldsymbol{\sigma}}=-\bar{p} \boldsymbol{i}+\overline{\boldsymbol{s}}$, where $\bar{p}$ is the pressure and $\overline{\boldsymbol{s}}$ the deviatoric stress. The stress in the pores being zero, the average stress in the matrix is $\frac{1}{1-c}(-\bar{p} \boldsymbol{i}+\overline{\boldsymbol{s}})$. If Eq. (46) holds and if the matrix is incompressible, the average strain in the matrix $\boldsymbol{M}$ is given by

$$
\langle\boldsymbol{\varepsilon}\rangle_{M}=\frac{\mathrm{d} u_{M}}{\mathrm{~d} \boldsymbol{\sigma}}\left(\frac{1}{1-c} \overline{\boldsymbol{s}}\right)
$$

and does not depend on the overall pressure. The average deformation of the pores depends on which model is used to describe the porous material. But all the above-mentioned models make use of some linear porous comparison material, the matrix of which having the secant, tangent or affine properties of the nonlinear matrix at the stress $\frac{1}{1-c} \bar{s}$, which again does not depend on the overall pressure. As a consequence, the deformation of the pores at fixed $\bar{s}$ will depend linearly on the pressure and so will the overall strain.

The true nonlinear behaviour of such materials is due to the heterogeneity of the local stress field in the matrix which locally might be deviatoric even under pure overall hydrostatic stress and thus involve the constitutive nonlinearities. Such intraphase heterogeneities are overlooked by models that deal only with per phase average quantities, which are therefore unable to describe the nonlinear answer of such materials under hydrostatic load.

\subsection{Two-phase isotropic incompressible composites}

We conclude this section with a comparison between the affine and secondorder procedures and several other schemes, for a two-phase composite made of incompressible isotropic power-law type phases, with local potentials

$$
w_{s}(\boldsymbol{\varepsilon})=\frac{\sigma_{s} \varepsilon_{0}}{m+1}\left(\frac{\varepsilon^{\mathrm{eq}}}{\varepsilon_{0}}\right)^{m+1}
$$

for purely deviatoric strain $\quad\left(w_{s}(\boldsymbol{\varepsilon})=+\infty\right.$ when $\operatorname{tr} \boldsymbol{\varepsilon} \neq 0$ to enforce 
incompressibility). Here, $\varepsilon_{0}$ is a reference strain and $\sigma_{s}$ characterises the stiffness of the phase $s$. The deviatoric stress and the restriction to purely deviatoric strains of the tensor of tangent moduli at some traceless reference strain $\boldsymbol{\varepsilon}_{s}$ are:

$$
\boldsymbol{\sigma}_{s}=2 \mu_{s}^{\mathrm{sct}}\left(\varepsilon_{s}^{\mathrm{eq}}\right) \boldsymbol{\varepsilon}_{s} \quad \text { and } \boldsymbol{L}_{s}=2 \mu_{s}^{\mathrm{tgt}}\left(\varepsilon_{s}^{\mathrm{eq}}\right) \boldsymbol{E}_{s}+2 \mu_{s}^{\mathrm{sct}}\left(\varepsilon_{s}^{\mathrm{eq}}\right) \boldsymbol{F}_{s}
$$

where $\mu_{s}^{\mathrm{tgt}}\left(\varepsilon_{s}^{\mathrm{eq}}\right)=\frac{m \sigma_{s}}{3 \varepsilon_{0}}\left(\frac{\varepsilon^{\mathrm{eq}}}{\varepsilon_{0}}\right)^{m-1}$ is the tangent modulus, $\mu_{s}^{\mathrm{sct}}\left(\varepsilon_{s}^{\mathrm{eq}}\right)=n \mu_{s}^{\mathrm{tgt}}\left(\varepsilon_{s}^{\mathrm{eq}}\right)$ is the secant modulus, $\boldsymbol{E}_{s}=\frac{2}{3} \frac{\boldsymbol{\varepsilon}_{s}}{\varepsilon_{s}} \otimes \frac{\boldsymbol{\varepsilon}_{s}}{\varepsilon_{c}}$ is the projector on the 'direction of the reference strain' and $\boldsymbol{F}_{s}=\boldsymbol{K}-\boldsymbol{E}_{s}, \boldsymbol{K}$ is the projector on purely deviatoric tensors.

The comparison is carried out for matrix-inclusion type composites with isotropically distributed spherical inclusions at low volume fraction, so that the classical Hashin-Shtrikman estimate built with the matrix as reference medium is an appropriate model for the description of the thermoelastic comparison composite. The relations given in Section 2 lead to the following system of equations characterising the deviatoric reference strains $\boldsymbol{\varepsilon}_{1}$ and $\boldsymbol{\varepsilon}_{2}$ for a given load $\bar{\varepsilon}$ :

$$
\begin{aligned}
& 2 \mu_{1}^{\mathrm{sct}}\left(\varepsilon_{1}^{\mathrm{eq}}\right) \boldsymbol{\varepsilon}_{1}+\boldsymbol{L}_{1}^{*}\left(\boldsymbol{L}_{1}\right): \boldsymbol{\varepsilon}_{1}=2 \mu_{2}^{\mathrm{sct}}\left(\varepsilon_{2}^{\mathrm{eq}}\right) \boldsymbol{\varepsilon}_{2}+\boldsymbol{L}_{1}^{*}\left(\boldsymbol{L}_{1}\right): \boldsymbol{\varepsilon}_{2} \\
& \boldsymbol{L}_{1}=2 \mu_{s}^{\mathrm{tgt}}\left(\varepsilon_{1}^{\mathrm{eq}}\right) \boldsymbol{E}_{1}+2 \mu_{s}^{\mathrm{sct}}\left(\varepsilon_{1}^{\mathrm{eq}}\right) \boldsymbol{F}_{1}, \quad c_{1} \boldsymbol{\varepsilon}_{1}+c_{2} \boldsymbol{\varepsilon}_{2}=\overline{\boldsymbol{\varepsilon}} .
\end{aligned}
$$

The constraint tensor $\boldsymbol{L}_{1}^{*}$ related to $\boldsymbol{L}_{1}$ of the form (49) has orthotropic symmetry and its full computation is in general a difficult task which has to be performed numerically. But when $\boldsymbol{\varepsilon}_{1}$ is a (traceless) uniaxial tension or a pure shear, it can be given the following expression (Nebozhyn and Ponte Castañeda, 2000)

$$
\boldsymbol{L}_{1}^{*}=2\left(\frac{1+n}{2 C(m, \theta)}-1\right) \mu_{1}^{\operatorname{tgt}} \boldsymbol{E}_{1}+\boldsymbol{G}_{1}
$$

where $\boldsymbol{G}_{1}$ satisfies $\boldsymbol{G}_{1}: \boldsymbol{E}_{1}=\boldsymbol{E}_{1}: \boldsymbol{G}_{1}=0$ and $C$ is the coefficient introduced by Suquet and Ponte Castañeda (1993), which depends on $m$ and the angle $\theta$ related to the third invariant of the tensor $\boldsymbol{\varepsilon}_{1}(\theta=0$ and $\theta=\pi / 6$ correspond to uniaxial tension and pure shear, respectively). Furthermore, for these specific values of $\theta$, it can be shown that the reference strains are proportional to the macroscopic strain, so that the system (50) can be simplified into a system of scalar equations characterising the equivalent strains $\varepsilon_{1}^{\mathrm{eq}}$ and $\varepsilon_{2}^{\mathrm{eq}}$, which is easy to solve numerically. The affine estimate for the overall stress is then obtained by averaging the stresses in the phases while the second order estimate (strain energy expansion) is computed according to Eq. (32); the dual variant of Eq. (32) leads to the effective complementary energy according to the stress energy expansion-based secondorder procedure. A macroscopic reference stress $\tilde{\boldsymbol{\sigma}}$ such that $\bar{\sigma}^{\mathrm{eq}}=\tilde{\sigma}\left(\bar{\varepsilon}^{\mathrm{eq}} / \varepsilon_{0}\right)^{m}$ (for the affine procedure) or $\bar{w}(\overline{\boldsymbol{\varepsilon}})=\frac{\tilde{\sigma} \varepsilon_{0}}{m+1}\left(\bar{\varepsilon}^{\mathrm{eq}} / \varepsilon_{0}\right)^{m+1}$ (for the second-order procedure) can then be identified.

The results are plotted in Fig. 2 as a function of the nonlinearity for a composite with $15 \%$ inclusions three times stiffer than the matrix and are compared to the predictions of the incremental, secant and variational estimates 


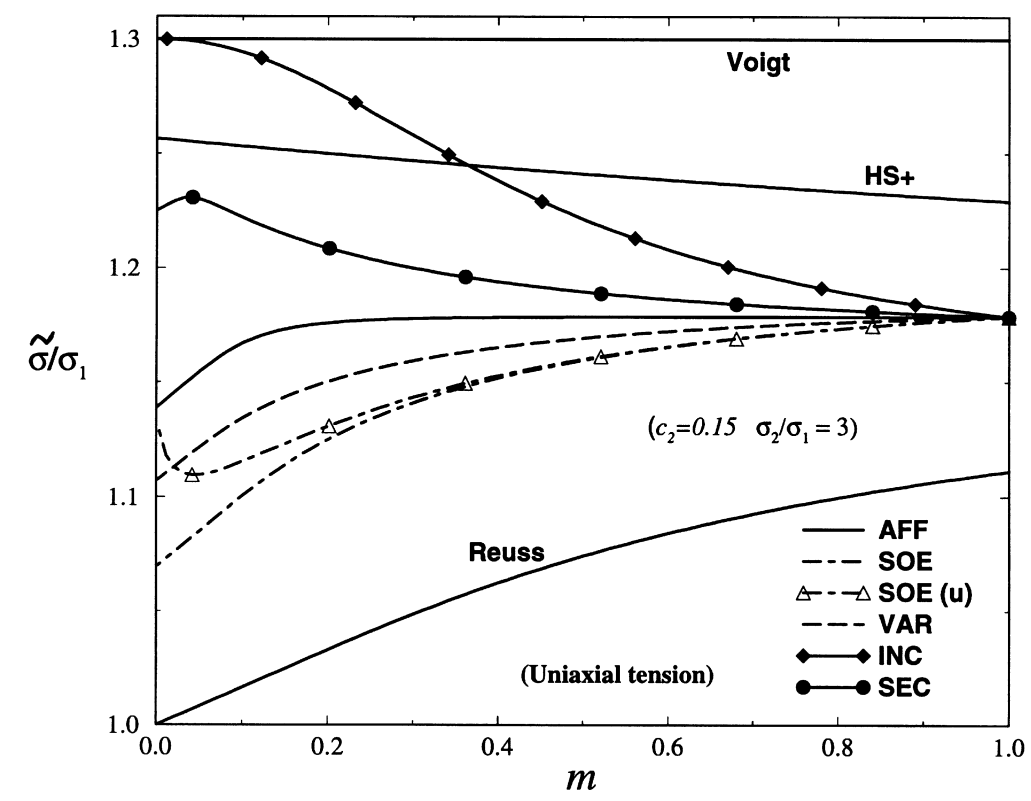

(a)

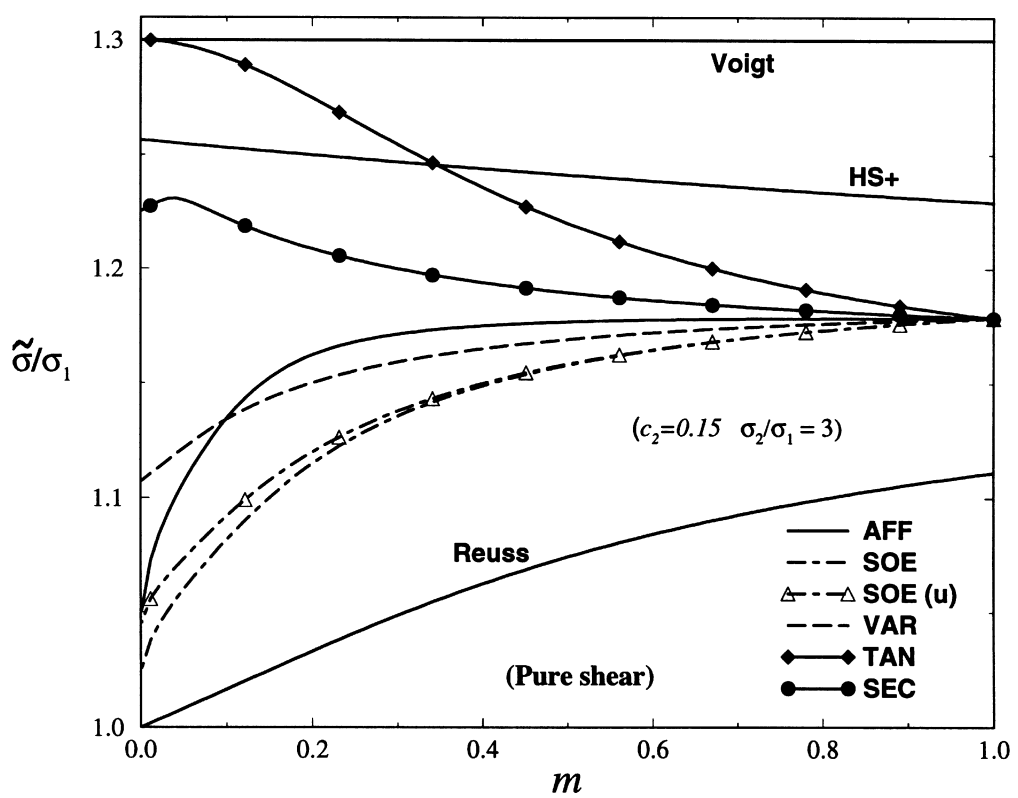

(b)

Fig. 2. Reference stress of a two-phase, incompressible, power-law type, matrix-inclusions composite, with $15 \%$ inclusions and a contrast of 3 , as a function of the nonlinearity $\mathrm{m}$. Predictions of the affine (AFF), second-order (SOE and SOE $(u)$ ), variational (VAR), incremental (INC), and secant (SEC) procedures combined with the Hashin-Shtrikman linear estimate, as well as rigourous Voigt, Reuss and upper Hashin-Shtrikman variational bounds are plotted, for uniaxial tension (a) and pure shear (b) Note that the bounds, the variational and the secant estimates, which are not sensitive to the third invariant of the load, are identical on both plots. 
as well as to the Voigt, Reuss and upper Hashin-Shtrikman variational bounds. It turns out that the affine estimate is the softest among all those dealing with phaseaveraged quantities; in particular, it improves substantially on the incremental procedure which is definitely too stiff at high nonlinearities. Unlike the classical or modified (i.e., variational) secant estimates, it is sensitive to the third invariant, as expected.

However, the affine estimate is stiffer than the variational one, over the whole range of nonlinearities in the case of tension as well as for $m>0.1$ for shear. Since the variational estimate is an upper bound for the effective nonlinear properties for the class of microstructures that can be described by the linear Hashin-Shtrikman estimates at any contrast of the phases, it is suspected that the affine estimate is still too stiff. Furthermore, there are situations where the affine estimate violates the variational Hashin-Shtrikman upper bound, which is valid for any microstructure with an isotropic distribution of the phases.

This occurs, in particular, for the case of small contrast and high nonlinearity. Indeed the small-contrast expansions (43) and (45) can be given explicit expressions in the present situation. Assuming overall isotropy for the composite, the effective stress-strain relation can be conveniently expressed in terms of the above-defined reference stress $\tilde{\sigma}$. The exact expansion (43) simplifies to

$$
\tilde{\sigma}^{\mathrm{SOE}}=\langle\sigma\rangle-C(m, \theta) \frac{\left\langle\sigma^{2}-\langle\sigma\rangle^{2}\right.}{\sigma_{0}}+O\left(t^{3}\right) .
$$

The expansion to second-order (45) of the affine method is made explicit by using the relations $\left\langle\dot{\boldsymbol{\varepsilon}}_{0}\right\rangle_{s}=-\left(\boldsymbol{L}_{0}^{*}+\boldsymbol{L}_{0}\right)^{-1}: \boldsymbol{\tau}_{s}$ together with the expression (51) of the constraint tensor $\boldsymbol{L}_{0}^{*}$ (valid at least when $\theta=0$ and $\theta=\pi / 6$ ). The result is

$$
\tilde{\sigma}^{\mathrm{AFF}}=\langle\sigma\rangle-\frac{2 m}{m+1}\left(1-\frac{m-1}{m+1} C(m, \theta)\right) C(m, \theta) \frac{\left\langle\sigma^{2}-\langle\sigma\rangle^{2}\right.}{\sigma_{0}}+O\left(t^{3}\right)
$$

and is different from the exact expression (52), except in the linear case $(m=1)$. In particular, when $m$ tends to $0, m C(m, \theta)$ tends to 0 and the prediction of the affine procedure tends (to second-order) to the Voigt upper bound $\tilde{\sigma}^{\mathrm{V}}=\langle\sigma\rangle$.

\section{Extension of thre affine formulation to rate-dependent and rate-independent elastoplasticity}

\subsection{Principle}

The affine formulation has been presented in Section 2 for nonlinear elasticity or viscosity for the sake of clarity. It can be extended to constitutive behaviours which exhibit a dependence of the current response on the loading path. Actually, a first draft of the affine method was originally proposed (Rougier et al., 1994) for rate-dependent elastoplasticity and we refer to Masson and Zaoui (1999) for its 
fully developed version. In this section, after a brief description of the general way to deal with hereditary behaviours, rate-independent elastoplasticity will be focused on, with special emphasis on the self-consistent scheme in view of comparison with the incremental formulation. Here, $\boldsymbol{\varepsilon}$ and $\boldsymbol{\gamma}$ again denote strains and shear strains and their rates are $\dot{\boldsymbol{\varepsilon}}$ and $\dot{\gamma}$.

The main difficulty is the need to account for the previous local and global responses at times $t \leq \tau$ when deriving the current response at $\tau$. Let $\overline{\boldsymbol{\varepsilon}}(t)$ be the prescribed macroscopic loading path, assumed to be zero for $t \leq 0$; we are searching for the macroscopic stress response $\overline{\boldsymbol{\sigma}}(\tau)$. According to the affine procedure, the resolution of an implicit set of equations for the local variables $\boldsymbol{\varepsilon}_{s}(\tau)$ or $\boldsymbol{\sigma}_{s}(\tau)$ is required. In addition, due to the considered hereditary behaviour, the knowledge of $\boldsymbol{\varepsilon}_{s}(t)$ or $\boldsymbol{\sigma}_{s}(t), \forall t \leq \tau$ is also required: these quantities must have been determined through a similar implicit treatment, involving at any time $t$ a specific linear comparison composite as defined at that time. Note that, in turn, each of these auxiliary problems at any intermediate time requires the knowledge of the local variables at all previous times. This results in a multiply implicit set of simultaneous equations which can only be solved numerically.

For rate-dependent elastoplasticity, it has been proposed (Masson and Zaoui, 1999) to discretise the time interval $[0, \tau]$ into intermediate times $\theta_{i}, i=1, N$ and to determine the behaviour of the linear (thermoviscoelastic) comparison composite and the associated local variables by proceeding through increasing times $\theta_{i}$, so as to use at each step the results obtained at previous steps. Despite the apparent similarity, this treatment is quite different from an incremental one which would not use the same linear comparison composite. The same difference will be stressed for rate-independent elastoplasticity for which a slightly modified method has to be proposed to deal with the specific situation of multibranched constitutive equations.

\subsection{Rate-independent elastoplasticity of crystalline materials}

We consider an elastoplastic polycrystal whose crystals obey the Schmid law with a limited number of definite crystallographic slip systems $\alpha$. The local strain rate is decomposed into its elastic and plastic parts:

$$
\dot{\boldsymbol{\varepsilon}}=\dot{\boldsymbol{\varepsilon}}^{\mathrm{e}}+\dot{\boldsymbol{\varepsilon}}^{\mathrm{p}}=\boldsymbol{S}: \dot{\boldsymbol{\sigma}}+\sum_{\alpha} \dot{\gamma}^{\alpha} \boldsymbol{R}^{\alpha},
$$

with $\boldsymbol{S}$ the elastic compliance, $\boldsymbol{R}^{\alpha}$ the orientation tensor (20) of system $\alpha$ and $\dot{\gamma}^{\alpha}$ the associated nonnegative plastic shear strain rate (see Section 2.4.2). A slip system $\alpha$ in phase $s$ is potentially active when its resolved shear stress $\boldsymbol{R}^{\alpha}: \boldsymbol{\sigma}_{s}$ reaches the critical value $\tau_{c}^{\alpha}$; it is active when, in addition, its rate $\boldsymbol{R}^{\alpha}: \dot{\boldsymbol{\sigma}}_{s}$ equals the critical resolved shear stress rate $\dot{\tau}_{c}^{\alpha}$, obeying the strain-hardening equation

$$
\dot{\tau}_{c}^{\alpha}=\sum_{\beta} H^{\alpha \beta} \dot{\gamma}^{\beta} .
$$


For simplicity, the hardening matrix $H^{\alpha \beta}$ is assumed to be symmetric, positive definite and constant (otherwise it would have to be linearised as well) so that the local (elastoplastic) tangent moduli take the form (Hutchinson, 1970\}:

$$
\left.\boldsymbol{L}_{s}=\boldsymbol{C}_{s}: \boldsymbol{I}-\sum_{\alpha \beta} D^{\alpha \beta} \boldsymbol{R}^{\alpha} \otimes \boldsymbol{R}^{\beta}: \boldsymbol{C}_{s}\right)
$$

where the summation is restricted to the active systems, with $\boldsymbol{C}_{s}$ the elastic moduli and $\boldsymbol{D}$ a matrix with components $D^{\alpha \beta}=\left(H^{\alpha \beta}+\boldsymbol{R}^{\alpha}: \boldsymbol{C}_{s}: \boldsymbol{R}^{\beta}\right)^{-1}$. The shear rate on active systems reads

$$
\gamma^{\alpha}=\sum_{\beta} D^{\alpha \beta} \boldsymbol{R}^{\beta}: \boldsymbol{C}_{s}: \dot{\boldsymbol{\varepsilon}}_{s}
$$

The tangent moduli $\boldsymbol{L}_{s}$, which have diagonal symmetry, depend on the current combination of active systems and are then multibranched. The intermediate times $\theta_{i}$ are defined as those times at which any new slip system in any phase of the polycrystal becomes active or inactive, an occurrence we call an event. Between two such consecutive events, the tangent moduli are, according to Eq. (56), constant throughout the polycrystal and the local constitutive equations may be linearised in the usual affine way: $\boldsymbol{\sigma}=\boldsymbol{L}_{s}: \boldsymbol{\varepsilon}+\boldsymbol{\tau}_{s}$, with $\boldsymbol{L}_{s}$ given by Eq. (56) and $\boldsymbol{\tau}_{s}=$ $\boldsymbol{\sigma}_{s}-\boldsymbol{L}_{s}: \boldsymbol{\varepsilon}_{s}$. The new difficulty of having to look at any time for the next event is somewhat balanced by an easier, stepwise explicit, solution procedure. On the other hand, the assumption of piecewise uniformity of the linear comparison medium is likely to be still cruder than it would be for smoother constitutive behaviour since, in the actual polycrystal, any new event occurring at some point makes the local instantaneous moduli change abruptly.

The solution scheme we propose to predict the overall stress response at time $\tau$ to a prescribed strain path $\overline{\boldsymbol{\varepsilon}}(t), t \leq \tau$, is the following:

- assume temporarily that at time $\tau$ the instant $\theta_{N}$ when the nearest previous event occurred is known as well as the average stress and strain tensors $\boldsymbol{\sigma}_{S N}$ and $\boldsymbol{\varepsilon}_{s N}$ at $t=\theta_{N}$ for all the phases and the slip amounts $\gamma_{N}^{\alpha}$ on all the slip systems;

- determine all the slip systems which are potentially active at $t=\theta_{N}$ throughout the polycrystal. From that, consider all the possible combinations of active systems in the polycrystal, each of them defining for the polycrystal a tentative branch;

- for each trial branch $(i)$, determine the local moduli $\boldsymbol{L}_{s}^{(i)}$ by Eq. (56) and prestress $\boldsymbol{\tau}_{s}^{(i)}=\boldsymbol{\sigma}_{s N}-\boldsymbol{L}_{s}^{(i)}: \boldsymbol{\varepsilon}_{s N}$ from $\boldsymbol{\sigma}_{s N}$ and $\boldsymbol{\varepsilon}_{s N}$ : these quantities remain constant as long as no new event occurs and they allow us to define the corresponding linear (thermoelastic) comparison composite and its overall characteristics $\tilde{\boldsymbol{L}}^{(i)}$ and $\tilde{\tau}^{(i)}$; by use of the associated localisation equations, derive updated local average strain (and then stress) tensors from the prescribed macroscopic strain, which yield the evolution of both the resolved and the critical shear stresses on all the slip systems (through Eqs. (55) and (57)) and then, by zeroing all the 
quantities $\left[\tau_{c}^{\alpha(i)}-\tau^{\alpha(i)}\right]$, the time $\theta_{N+1}^{(i)}$ of the occurrence of the next nearest event;

- the consistency of the trial branch (i) can then be checked, i.e., the satisfaction at time $\theta_{N+1}^{(i)}$ of the plastic criterion for the systems which had been considered as active as well as inactive at time $\theta_{N}$. This can be done by starting, for the active systems, from the whole set of potentially active systems at $\theta_{N}$ and by successively suppressing any one, or two, or more systems and applying the same procedure as before until consistency is satisfied. When reached, it yields the expected response $\overline{\boldsymbol{\sigma}}(\tau)$ as well as the local states at $\theta_{N+1}^{(i)}$;

- since $\theta_{N}, \boldsymbol{\sigma}_{S N}, \boldsymbol{\varepsilon}_{S N}$ and $\gamma_{N}^{\alpha}$ are not known, proceed by increasing intermediate times from $t=0$ and follow the above procedure from $\theta_{1}$ to $\theta_{N}$, so as to use at each intermediate time $\theta_{i}$ the initial quantities which have been derived before.

\subsection{Comments and illustration}

The affine treatment must be clearly distinguished from the incremental one. This can be checked for the self-consistent scheme by comparing the localisation equations at two successive times $\theta_{i}$ and $\theta_{i+1}$. From the dual variant of Eq. (7), specified for the self-consistent scheme, we can write with simplified notations:

$$
\boldsymbol{\varepsilon}_{s}\left(\theta_{i}\right)=\boldsymbol{A}_{s}^{(i)}:\left[\overline{\boldsymbol{\varepsilon}}\left(\theta_{i}\right)+\boldsymbol{P}^{(i)}:\left(\tilde{\boldsymbol{\tau}}^{(i)}-\boldsymbol{\tau}_{s}^{(i)}\right)\right] ; \quad \boldsymbol{A}_{s}^{(i)}=\left(\boldsymbol{I}+\boldsymbol{P}^{(i)}: \boldsymbol{\delta} \boldsymbol{L}_{s}^{(i)}\right)^{-1}
$$

where the exponent $(i)$ refers to the comparison composite at time $\theta_{i}$. Similar relations can be written at time $\theta_{i+1}$ but involve another comparison composite. When $\theta_{i+1}$ is sufficiently close to $\theta_{i}$, the variation $\Delta \boldsymbol{\varepsilon}_{s}\left(\theta_{i}\right)$ of the local strain is

$$
\Delta \varepsilon_{s}\left(\theta_{i}\right) \approx \boldsymbol{A}_{s}^{(i)}: \Delta \overline{\boldsymbol{\varepsilon}}\left(\theta_{i}\right)+\Delta^{(i)} \boldsymbol{A}_{s}: \overline{\boldsymbol{\varepsilon}}\left(\theta_{i}\right)+\Delta^{(i)}\left[\boldsymbol{A}_{s}: \boldsymbol{P}:\left(\tilde{\boldsymbol{\tau}}-\boldsymbol{\tau}_{s}\right)\right]
$$

where $\Delta^{(i)} X=X^{(i+1)}-X^{(i)}$. This expression, which is a transcription of the time derivative of the linear thermoelastic localisation equation, takes into account the modification of the comparison medium associated with the next event. In the same situation, Hill's incremental expression reduces to $\Delta \boldsymbol{\varepsilon}_{s}\left(\theta_{i}\right)=\boldsymbol{A}_{s}^{(i)}: \Delta \bar{\varepsilon}\left(\theta_{i}\right)$.

So, even with equal $\boldsymbol{A}_{s}^{(i)}$ values, the localisation equations are different. For example, the first step of a tensile response is elastic, so that the stress and strain per phase averages and the initial time $\theta_{1}$ are the same for both formulations: the first branch is identical beyond $\theta_{1}$, with identical resulting tangent moduli and strain localisation tensors, but the local strain rates will slightly differ and the overall estimates will gradually deviate from each other. Fig. 3 provides an illustration of this difference: the tensile stress-strain curves of an untextured fcc polycrystal without intracrystalline hardening as predicted from the incremental (Hutchinson, 1970) and affine formulations of the self-consistent scheme are compared. Again the affine treatment yields softer predictions than Hill's one. 


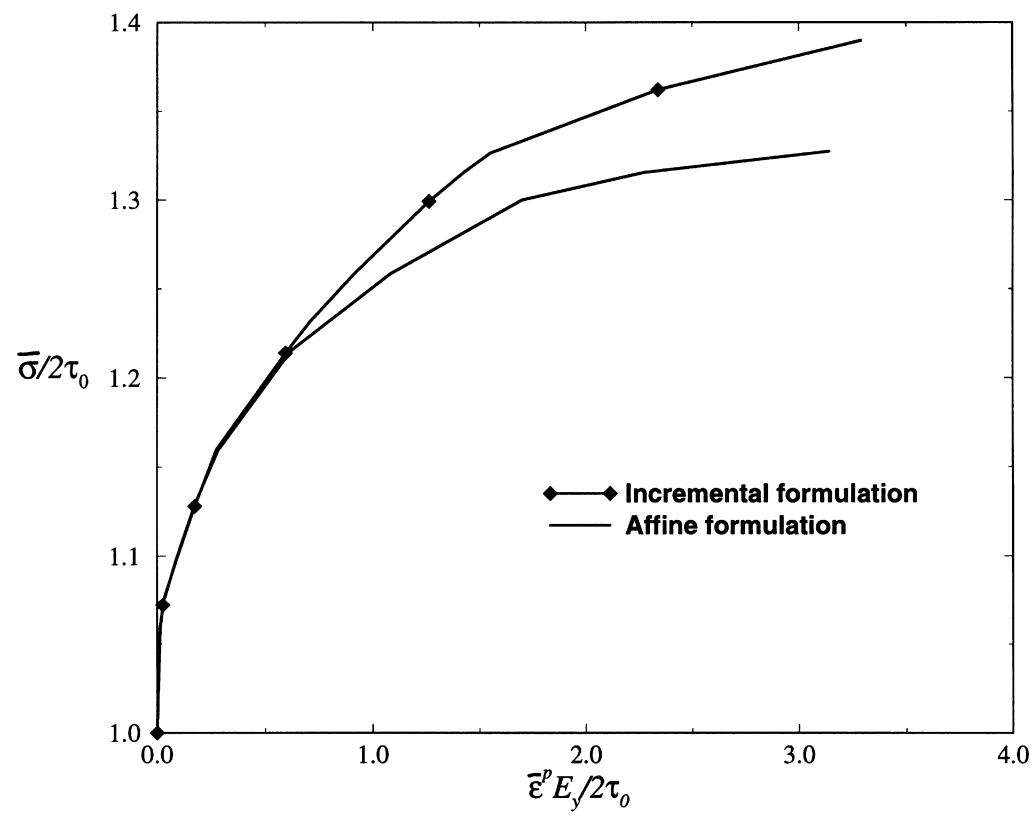

Fig. 3. Tensile stress-plastic strain curves of an untextured fcc polycrystal according to the incremental and affine versions of the self-consistent scheme (isotropic elasticity with Young's modulus $E_{\mathrm{y}}$ and Poisson's ratio $v=1 / 3$; non-hardening single crystals with $\tau_{c}=\tau_{0}$ ).

\section{Conclusion}

The affine formulation offers an improved alternative to Hill's incremental (as well as to the tangent) formulation to estimate the effective properties of nonlinear heterogeneous materials for both hereditary and non-hereditary constitutive behaviours and without restrictions on the specific form of the constitutive equations. However, it has been shown to display definite limitations which are likely to originate from the identification of the reference strain or stress tensors with the per phase average ones. When compared with the second-order procedure, which relies on the same assumption, it turns out that, except for the duality gap, the second-order description based on potentials offers marked advantages for nonlinear elasticity and viscoplasticity; on the other hand, the direct stress-strain approach of the affine formulation makes it possible to explore the field of hereditary constitutive behaviours, both for rate-dependent and rateindependent elastoplasticity.

There are still open questions mainly pertaining to the choice of the 'best' thermoelastic comparison composite and of possibly improved reference stresses or strains. There is a need to improve both the classical affine and second-order estimates, regarding the existence of an overall potential, the resolution of the 
duality gap, the consistency to small contrast expansion and variational bounds and the response of porous materials.

\section{Acknowledgements}

This study is part of the program Micromechanics of nonlinear composites and polycrystals supported by NSF (USA) and CNRS (France). Fruitful discussions with P. Ponte Castañeda, partner of this program, are gratefully acknowledged.

\section{References}

Berveiller, M., Zaoui, A., 1979. An extension of the self-consistent scheme to plastically-flowing polycrystals. J. Mech. Phys. Solids 26, 325-344.

Buryachenko, V., 1996. The overall elastoplastic behavior of multiphase materials with isotropic components. Acta Mechanica 119, 93-117.

Gilormini, P. 1996. A critical evaluation of various nonlinear extensions of the self-consistent model. In: Pineau, A., Zaoui, A. (Eds.), Micromechanics of Plasticity and Damage of Multiphase Materials. Kluwer Academic Publishers, Dordrecht, The Netherlands, pp. 67-74.

Hill, R., 1965. Continuum micro-mechanics of elastoplastic polycrystals. J. Mech. Phys. Solids 13, 89101.

Hu, G., 1996. A method of plasticity for general aligned spheroidal void of fiber-reinforced composites. Int. J. Plasticity 12, 439-449.

Hutchinson, J.W., 1970. Elastic-plastic behaviour of polycrystalline metals and composites. Proc. R. Soc. Lond. A319, 247-272.

Hutchinson, J.W., 1976. Bounds and self-consistent estimates for creep of polycrystalline materials. Proc. R. Soc. Lond. A348, 101-127.

Kröner, E., 1961. Zur plastischen Verformung des Vielkristalls. Acta Metall. 9, 155-161.

Lebensohn, R., Tomé, C.N., 1993. A self-consistent anisotropic approach for the simulation of plastic deformation and texture development of polycrystals: application to zirconium alloys. Acta Metall. Mater. 41, 2611-2624.

Levin, V.M., 1967. Thermal expansion coefficients of heterogeneous materials. Mekh. Tverd. Tela 2 (8), 38-94.

Masson, R., Zaoui, A., 1999. Self-consistent estimates for the rate-dependent elastoplastic behaviour of polycrystalline materials. J. Mech. Phys. Solids 47, 1543-1568.

Molinari, A., Canova, G.R., Ahzi, S., 1987. A self-consistent approach of the large deformation polycrystal viscoplasticity. Acta Metall. 35 (12), 2983-2994.

Nebozhyn, M.V., Ponte Castañeda, P., 2000. The second-order procedure: corrected results for isotropic, two-phase composites. J. Mech. Phys. Solids, submitted.

Ponte Castañeda, P., 1991. The effective mechanical properties of nonlinear isotropic composites. J. Mech. Phys. Solids 39 (1), 45-71.

Ponte Castañeda, P., 1996. Exact second-order estimates for the effective mechanical properties of nonlinear composite materials. J. Mech. Phys. Solids 44 (6), 827-862.

Ponte Castañeda, P., Suquet, P., 1998. Nonlinear composites. Adv. Appl. Mech. 34, 171-302.

Qiu, Y.P., Weng, G.J., 1992. A theory of plasticity for porous materials and particle-reinforced composites. J. Appl. Mech. 59, 261-268.

Rougier, Y., Stolz, C., Zaoui, A., 1994. Self-consistent modelling of elastic-viscoplastic polycrystals. C. R. Acad. Sci. Paris, Série II 318, 145-151.

Suquet, P., 1993. Overall potentials and extremal surfaces of power law or ideally plastic materials. J. Mech. Phys. Solids 41, 981-1002. 
Suquet, P., 1995. Overall properties of nonlinear composites: a modified secant moduli theory and its link with Ponte Castañeda's nonlinear variational procedure. C. R. Acad. Sci. Paris, Série II 320 (b), 563-571.

Suquet, P., 1997. Effective properties of nonlinear composites. Continuum Micromechanics. In: Suquet, P. (Ed.), CISM Lecture Notes, vol. 377. Springer-Verlag, Berlin, pp. 197-264.

Suquet, P., Ponte Castañeda, P., 1993. Small-contrast perturbation expansions for the effective properties of nonlinear composites. C. R. Acad. Sci. Paris, Série II 317, 1515-1522.

Talbot, D.R.S., Willis, J.R., 1985. Variational principles for inhomogeneous nonlinear media. IMA J. Appl. Math. 35, 39-54.

Tandon, G.P., Weng, G.J., 1988. A theory of particle-reinforced plasticity. J. Appl. Mech. 55 (1), 126135.

Taylor, G.I., 1938. Plastic strain in metals. J. Inst. Metals 62, 307-324.

Willis, J.R., 1977. Bounds and self-consistent estimates for the overall moduli of anisotropic composites. J. Mech. Phys. Solids 25, 185-202.

Willis, J.R., 1981. Variational and related methods for the overall properties of composites. Adv. Appl. Mech. 21, 1-78.

Willis, J.R., 1983. The overall response of composite materials. J. Appl. Mech. 50, 1202-1209. 